\title{
Nonlinear System Stabilization via Hierarchical Switching Control
}

\author{
Alexander Leonessa, Wassim M. Haddad, and VijaySekhar Chellaboina
}

\begin{abstract}
In this paper, a nonlinear control-system design framework predicated on a hierarchical switching controller architecture parameterized over a set of moving system equilibria is developed. Specifically, using equilibria-dependent Lyapunov functions, a hierarchical nonlinear control strategy is developed that stabilizes a given nonlinear system by stabilizing a collection of nonlinear controlled subsystems. The switching nonlinear controller architecture is designed based on a generalized lower semicontinuous Lyapunov function obtained by minimizing a potential function over a given switching set induced by the parameterized system equilibria. The proposed framework provides a rigorous alternative to designing gain-scheduled feedback controllers and guarantees local and global closed-loop system stability for general nonlinear systems.
\end{abstract}

Index Terms-Domains of attraction, dynamic compensation, equilibria-dependent Lyapunov functions, hierarchical switching control, nonlinear connective stabilization, parameterized equilibria.

\section{INTRODUCTION}

$\mathbf{I}$ F THE operating range of the control system is small, and if the system nonlinearities are smooth, then the control system can be locally approximated by a linearized system around a given operating condition and linear multivariable control theory can then be used to maintain local stability and performance. However, in high-performance engineering applications, such as advanced tactical fighter aircraft and variable-cycle gas turbine aeroengines, the locally approximated linearized system does not cover the operating range of the system dynamics. In this case, gain-scheduled controllers can be designed over several fixed operating points covering the system's operating range and controller gains interpolated over this range [1], [2]. However, due to approximation linearization errors and neglected operating point transitions, the resulting gain-scheduled system does not have any guarantees of performance or stability. Even though stability properties of gain-scheduled controllers are analyzed in [3] and [4] and

Manuscript received September 18, 1998; revised June 24, 1999 and March 10, 2000. Recommended by Associate Editor A. Rantzer. This work was supported in part by the National Science Foundation under Grant ECS-9496249, the Air Force Office of Scientific Research under Grant F49620-001-0095, and the Army Research Office under Grant DAAH04-96-1-0008.

A. Leonessa is with the Department of Ocean Engineering, Florida Atlantic University-Sea Tech, Dania Beach, FL 33004-3023 USA (e-mail aleo@seatech.fau.edu)

W. M. Haddad is with the School of Aerospace Engineering, Georgia Institute of Technology, Atlanta, GA 30332-0150 USA (e-mail: wm.haddad@aerospace.gatech.edu)

V. S. Chellaboina is with Mechanical and Aerospace Engineering, University of Missouri, Columbia, MO 65211 USA (e-mail: ChellaboinaV@missouri.edu).

Publisher Item Identifier S 0018-9286(01)00033-2. stability guarantees are provided for plant output scheduling, a design framework for gain-scheduling control guaranteeing system stability over an operating range of the nonlinear plant dynamics has not been addressed in the literature.

In an attempt to develop a design framework for gain-scheduling control, linear parameter-varying system theory has been developed [5], [6]. Since gain-scheduling control involves a linear parameter-dependent plant, linear parameter-varying methods for gain scheduling seem natural. However, even though this is indeed the case for linear dynamical systems involving exogenous parameters, this is not the case for nonlinear dynamical systems. This is due to the fact that a nonlinear system cannot be represented as a true linear parameter-varying system since the varying system parameters are endogenous, i.e., functions of the system state. Hence, stability and performance guarantees of linear parameter-varying systems do not extend to the nonlinear system. Of course, in the case where the magnitude and rate of the endogenous parameters are constrained such that the linear parameter-varying system hopefully behaves closely to the actual nonlinear system, then stable controllers can be designed using quasilinear parameter-varying representations [7]. However, in the case of unexpectedly large amplitude uncertain exogenous disturbances and/or system parametric uncertainty, a priori assumptions on magnitude and rate constraints on endogenous parameters are unverifiable.

In this paper, a nonlinear control design framework predicated on a hierarchical switching controller architecture parameterized over a set of moving system equilibria is developed. Specifically, using equilibria-dependent Lyapunov functions or, instantaneous (with respect to a given parameterized equilibrium manifold) Lyapunov functions, a hierarchical nonlinear control strategy is developed that stabilizes a given nonlinear system using a supervisory switching controller that coordinates lower level stabilizing subcontrollers [8]-[10]. Each subcontroller can be nonlinear, and thus, local set point designs can be nonlinear. Furthermore, for each parameterized equilibrium manifold, the collection of subcontrollers provides guaranteed domains of attraction with nonempty intersections that cover the region of operation of the nonlinear system in the state space. A hierarchical switching nonlinear controller architecture is developed based on a generalized lower semicontinuous Lyapunov function obtained by minimizing a potential function, associated with each domain of attraction, over a given switching set induced by the parameterized system equilibria. The switching set specifies the subcontroller to be activated at the point of switching, which occurs within the intersections of the domains of attraction. The hierarchical switching nonlinear 
controller guarantees that the generalized Lyapunov function is nonincreasing along the closed-loop system trajectories with strictly decreasing values at the switching points, establishing asymptotic stability. In the case where one of the parameterized system equilibrium points is globally asymptotically stable, the proposed nonlinear stabilization framework guarantees global attraction to any given system invariant set. If, in addition, a structural topological constraint is enforced on the switching set, then the proposed framework guarantees global asymptotic stability of any given system equilibrium on the parameterized system equilibrium manifold. Furthermore, since the proposed switching nonlinear control strategy is predicted on a generalized Lyapunov function framework with strictly decreasing values at the switching points, the possibility of a sliding mode is precluded. Hence, the proposed nonlinear stabilization framework avoids the undesirable effects of high-speed switching onto an invariant sliding manifold, which is one of the main limitations of variable structure controllers. Finally, we note that the present framework provides a rigorous alternative to designing gain-scheduled controllers for general nonlinear systems by explicitly capturing plant nonlinearities and quantifing the notion of slow-varying system parameters which place fundamental limitations on achievable performance of gain-scheduling controllers.

Limited to system analysis, related yet different approaches to the proposed hierarchical switching control design framework are given in [11]-[14]. Specifically, analysis of switched linear systems in the plane $\left(\mathbb{R}^{2}\right)$ are given in [11] and [12]. More recently, asymptotic stability analysis of m-linear systems using Lyapunov-like functions is given in [13]. Stability of a multi-controller switched system is analyzed using Lyapunov functions and sliding surfaces in [14]. A hybrid stabilization strategy for nonlinear systems controlled by linear controllers is discussed in [15] wherein domains of attraction are enlarged by the use of a switching strategy. However, this analysis is limited to linearly controlled systems in the plane. Even though the approach can be extended to higher order systems, the computational complexity needed to analyze the direction of the closed-loop system flows render the approach impractical. The special issues on hybrid control systems, [16], [17] present an excellent analysis expansion on switching systems with controller design methods limited to specific applications. We note that the concept of equilibria-dependent Lyapunov functions was first introduced by the authors in [18] where a globally stabilizing control design framework for controlling rotating stall and surge in axial flow compressors was developed. In parallel research to [18], a related yet different approach was introduced in [19] wherein control Lyapunov functions for nonlinear systems linearized about a finite number of "trim" points to guarantee stability of a range of operating conditions were given. Specifically, in the case where we specialize the switching set to a finite number of equilibrium points, we recover the results of [19]. Finally, we emphasize that our approach is constructive in nature rather than existential. In particular, we provide an explicit construction for a hierarchical switching controller for nonlinear system stabilization and, in this case, our constructive conditions are complementary to existential results on asymptotic controllability via discontinuous feedback [20].

\section{Mathematical PRELIMINARIES}

In this section, we establish definitions, notation, and two key results used later in the paper. Let $\mathbb{R}$ denote the set of real numbers, let $\mathbb{R}^{n}$ denote the set of $n \times 1$ real column vectors, let $\mathbb{R}^{n \times m}$ denote the set of real $n \times m$ matrices, and let $(\cdot)^{T}$ denote transpose. Furthermore, we write $\|\cdot\|$ for the Euclidean vector norm, $V^{\prime}(x)$ for the Fréchet derivative of $V(\cdot)$ at $x$, and $A \geq 0$ (resp., $A>0$ ) to denote the fact that the Hermitian matrix $A$ is nonnegative (resp., positive) definite. For a subset $\mathcal{S} \subset \mathbb{R}^{n}$, we write $\partial \mathcal{S}, \mathcal{S}, \overline{\mathcal{S}}$ for the boundary, the interior, and the closure of $\mathcal{S}$, respectively. Finally, let $C^{0}$ denote the set of continuous functions and $C^{r}$ denote the set of functions with $r$-continuous derivatives.

In this paper, we consider nonlinear controlled dynamical systems of the form

$$
\dot{x}(t)=F(x(t), u(t)), \quad x(0)=x_{0}, t \in \mathcal{I}_{x_{0}}
$$

where $x(t) \in \mathcal{D} \subseteq \mathbb{R}^{n}, t \in \mathcal{I}_{x_{0}}$, is the system state vector, $\mathcal{I}_{x_{0}} \subseteq \mathbb{R}$ is the maximal interval of existence of a solution $x(\cdot)$ of (1), $\mathcal{D}$ is an open set, $0 \in \mathcal{D}, u(t) \in \mathcal{U} \subseteq \mathbb{R}^{m}, t \in \mathcal{I}_{x_{0}}$, is the control input, $\mathcal{U}$ is the set of all admissible controls such that $u(\cdot)$ is a measurable function with $0 \in \mathcal{U}$, and $F: \mathcal{D} \times \mathcal{U} \rightarrow \mathbb{R}^{n}$ is continuous on $\mathcal{D} \times \mathcal{U}$.

Definition 2.1: The point $\bar{x} \in \mathcal{D}$ is an equilibrium point of (1) if there exists $\bar{u} \in \mathcal{U}$ such that $F(\bar{x}, \bar{u})=0$.

In this paper we assume that given an equilibrium point $\bar{x} \in$ $\mathcal{D}$ corresponding to $\bar{u} \in \mathcal{U}$ and a mapping $\varphi: \mathcal{D} \times \Lambda \rightarrow \mathcal{U}$, $\Lambda \subseteq \mathbb{R}^{q}, 0 \in \Lambda$, such that $\varphi(\bar{x}, 0)=\bar{u}$, there exist neighborhoods $\mathcal{D}_{o} \subset \mathcal{D}$ of $\bar{x}$ and $\Lambda_{o} \subset \Lambda$ of 0 , and a continuous function $\psi: \Lambda_{o} \rightarrow \mathcal{D}_{o}$ such that $\bar{x}=\psi(0)$, and, for every $\lambda \in \Lambda_{o}, x_{\lambda}=\psi(\lambda)$ is an equilibrium point; that is, $F(\psi(\lambda), \varphi(\psi(\lambda), \lambda))=0, \lambda \in \Lambda_{o}$. This is a necessary condition for parametric stability with respect to $\Lambda_{o}$ as defined in [21] and [22]. Note that the connected set $\Lambda \subseteq \mathbb{R}^{q}$ corresponds to a parameterization set with the function $\psi(\cdot)$ parameterizing the system equilibria. In the special case where $q=m$ and $\varphi(x, \lambda)=\lambda$, it follows that the parameterized system equilibria are given by the constant control $u(t) \equiv \lambda$. A parameterization that provides a local characterization of an equilibrium manifold, including in neighborhoods of bifurcation points, is given in [23]. Alternatively, the well-known implicit function theorem provides sufficient conditions for guaranteeing the existence of such a parameterization under the more restrictive condition of continuous differentiability of the mapping $\psi(\cdot)$.

Next, we consider nonlinear feedback controlled dynamical systems. A measurable mapping $\phi: \mathcal{D} \rightarrow \mathcal{U}$ satisfying $\phi(\bar{x})=\bar{u}$ is called at control law. Furthermore, if $u(t)=\phi(x(t))$, where $\phi(\cdot)$ is a control law and $x(t), t \in \mathcal{I}_{x_{0}}$, satisfies (1), then $u(\cdot)$ is called a feedback control law. Here, we consider nonlinear closed-loop dynamical systems of the form

$$
\dot{x}(t)=F(x(t), \phi(x(t))), \quad x(0)=x_{0}, \quad t \in \mathcal{I}_{x_{0}} .
$$

A function $x: \mathcal{I}_{x_{0}} \rightarrow \mathcal{D}$ is said to be a solution to (2) on the interval $\mathcal{I}_{x_{0}} \subseteq \mathbb{R}$ with initial condition $x(0)=x_{0}$, if $x(t)$ satisfies (2) for all $t \in \mathcal{I}_{x_{0}}$. Note that we do not assume any regularity condition on the function $\phi(\cdot)$. However, we do assume that for 
every $y \in \mathcal{D}$ there exists a unique solution $x(\cdot)$ of $(2)$ defined on $\mathcal{I}_{y}$ satisfying $x(0)=y$. Furthermore, we assume that all the solutions $x(t), t \in \mathcal{I}_{x_{0}}$, to (2) are continuous functions of the system initial conditions $x_{0} \in \mathcal{D}$ which, with the assumption of uniqueness of solutions, implies continuity of solutions $x(t)$, $t \in \mathcal{I}_{x_{0}}$, to (2) [24, p. 24].

Remark 2.1: If $F(\cdot, \phi(\cdot))$ is Lipschitz-continuous on $\mathcal{D}$, then there exists a unique solution to (2). In this case, the semi-group property $s\left(t+\tau, x_{0}\right)=s\left(t, s\left(\tau, x_{0}\right)\right), t, \tau \in \mathcal{I}_{x_{0}}$, and the continuity of $s(t, \cdot)$ on $\mathcal{D}, t \in \mathcal{I}_{x_{0}}$, hold, where $s\left(\cdot, x_{0}\right)$ denotes the solution of the nonlinear feedback controlled dynamical system (2). Alternatively, uniqueness of solutions in time along with the continuity of $F(\cdot, \phi(\cdot))$ ensure that the solutions to (2) satisfy the semi-group property and are continuous functions of the initial condition $x_{0} \in \mathcal{D}$ even when $F(\cdot, \phi(\cdot))$ is not Lipschitz-continuous on $\mathcal{D}$ (see [25, Th. 4.3, p. 59]). More generally, $F(\cdot, \phi(\cdot))$ need not to be continuous. In particular, if $F(\cdot, \phi(\cdot))$ is discontinuous but bounded, and $x(\cdot)$ is the unique solution to (2) in the sense of Filippov [26], then the semi-group property along with the continuous dependence of solutions on initial conditions hold [26].

Next, we introduce several definitions and key results that are necessary for the main results of this paper.

Definition 2.2: Let $\mathcal{D}_{c} \subseteq \mathcal{D}$ and let $V: \mathcal{D}_{c} \rightarrow \mathbb{R}$. For $\alpha \in \mathbb{R}$, the set $V^{-1}(\alpha) \triangleq\left\{x \in \mathcal{D}_{c}: V(x)=\alpha\right\}$ is called the $\alpha$-level set. For $\alpha, \beta \in \mathbb{R}, \alpha \leq \beta$, the set $V^{-1}([\alpha, \beta]) \triangleq\left\{x \in \mathcal{D}_{c}: \alpha \leq\right.$ $V(x) \leq \beta\}$ is called the $[\alpha, \beta]$-sublevel set.

Definition 2.3: A set $\mathcal{M}^{+} \subseteq \mathcal{D} \subseteq \mathbb{R}^{n}$ (resp., $\mathcal{M}^{-}$) is a positively (resp., negatively) invariant set for the nonlinear feedback controlled dynamical system (2) if $x_{0} \in \mathcal{M}^{+}$(resp., $\mathcal{M}^{-}$) implies that $[0,+\infty) \subseteq \mathcal{I}_{x_{0}}$ (resp., $\left.(-\infty, 0] \subseteq \mathcal{I}_{x_{0}}\right)$ and $x(t) \in$ $\mathcal{M}^{+}$(resp., $\mathcal{M}^{-}$) for all $t \geq 0$ (resp., $t \leq 0$ ). A set $\mathcal{M} \subseteq$ $\mathcal{D} \subseteq \mathbb{R}^{n}$ is an invariant set for the nonlinear feedback controlled dynamical system (2) if $x_{0} \in \mathcal{M}$ implies that $\mathcal{I}_{x_{0}}=\mathbb{R}$ and $x(t) \in \mathcal{M}$ for all $t \in \mathbb{R}$.

Definition 2.4: $p \in \overline{\mathcal{D}} \subseteq \mathbb{R}^{n}$ is a positive limit point of the trajectory $x(t), t \in \mathcal{I}_{x_{0}}$, if $[0,+\infty) \subseteq \mathcal{I}_{x_{0}}$ and there exists a sequence $\left\{t_{n}\right\}_{n=0}^{\infty}$, with $t_{n} \rightarrow \infty$ as $n \rightarrow \infty$, such that $x\left(t_{n}\right) \rightarrow$ $p$ as $n \rightarrow \infty$. The set of all positive limit points of $x(t), t \in \mathcal{I}_{x_{0}}$, is the positive limit set $\mathcal{P}_{x_{0}}^{+}$of $x(t), t \in \mathcal{I}_{x_{0}}$.

The following result on positive limit sets is fundamental and forms the basis for all the generalized stability and invariant set theorems developed in Section III.

Lemma 2.1 [27]: Suppose the forward solution $x(t), t \geq 0$, to (2) corresponding to an initial condition $x(0)=x_{0}$ exists and is bounded. Then the positive limit set $\mathcal{P}_{x_{0}}^{+}$of $x(t), t \in \mathcal{I}_{x_{0}}$, is a nonempty, compact, connected invariant set. Furthermore, $x(t) \rightarrow \mathcal{P}_{x_{0}}^{+}$as $t \rightarrow \infty$.

The following definition introduces three types of stability, as well as attraction of (2) with respect to a compact positively invariant set.

Definition 2.5: Let $\mathcal{D}_{0} \subset \mathcal{D}$ be a compact positively invariant set for the nonlinear feedback controlled dynamical system (2). $\mathcal{D}_{0}$ is Lyapunov stable if for every open neighborhood $\mathcal{O}_{1} \subseteq \mathcal{D}$ of $\mathcal{D}_{0}$, there exists an open neighborhood $\mathcal{O}_{2} \subseteq \mathcal{O}_{1}$ of $\mathcal{D}_{0}$ such that $x(t) \in \mathcal{O}_{1}, t \geq 0$, for all $x_{0} \in \mathcal{O}_{2}$. $\mathcal{D}_{0}$ is attractive if there exists an open neighborhood $\mathcal{O}_{3} \subseteq \mathcal{D}$ of $\mathcal{D}_{0}$ such that $\mathcal{P}_{x_{0}}^{+} \subseteq \mathcal{D}_{0}$ for all $x_{0} \in \mathcal{O}_{3}$. $\mathcal{D}_{0}$ is asymptotically stable if it is Lyapunov stable and attractive. $\mathcal{D}_{0}$ is globally asymptotically stable if it is Lyapunov-stable and $\mathcal{P}_{x_{0}}^{+} \subseteq \mathcal{D}_{0}$ for all $x_{0} \in \mathbb{R}^{n}$. Finally, $\mathcal{D}_{0}$ is unstable if it is not Lyapunov-stable.

Next, we give a set theoretic definition involving the domain, or region, of attraction of the compact positively invariant set $\mathcal{D}_{c}$ of (2).

Definition 2.6: Suppose the compact positively invariant set $\mathcal{D}_{0} \subset \mathcal{D}$ of (2) is attractive. Then the domain of attraction $\mathcal{D}_{A}$ of $\mathcal{D}_{0}$ is defined as $\mathcal{D}_{A} \triangleq\left\{x_{0} \in \mathcal{D}: \mathcal{P}_{x_{0}}^{+} \subseteq \mathcal{D}_{0}\right\}$.

Next, we present a key theorem due to Weierstrass involving lower semicontinuous functions on compact sets. For the statement of the result the following definition is needed.

Definition 2.7: Let $\mathcal{D}_{c} \subset \mathcal{D}$. A function $V: \mathcal{D}_{c} \rightarrow \mathbb{R}$ is lower semicontinuous on $\mathcal{D}_{c}$ if for every sequence $\left\{x_{n}\right\}_{n=0}^{\infty} \subset \mathcal{D}_{c}$ such that $\lim _{n \rightarrow \infty} x_{n}=x, V(x) \leq \liminf _{n \rightarrow \infty} V\left(x_{n}\right)$.

Theorem 2.1 [29]: Suppose $\mathcal{D}_{c} \subset \mathcal{D}$ is compact and $V: \mathcal{D}_{c} \rightarrow \mathbb{R}$ is lower semicontinuous. Then there exists $x^{*} \in \mathcal{D}_{c}$ such that $V\left(x^{*}\right) \leq V(x), x \in \mathcal{D}_{c}$

Finally, the following definition is used in the paper.

Definition 2.8: A function $V: \mathcal{D} \rightarrow \mathbb{R}$ is positive definite on $\mathcal{D} \backslash \mathcal{D}_{0}$, where $\mathcal{D}_{0} \subset \mathcal{D}$, if $V(x)=0, x \in \mathcal{D}_{0}$, and $V(x)>0$, $x \in \mathcal{D} \backslash \mathcal{D}_{0}$. A function $V: \mathcal{D} \rightarrow \mathbb{R}$ is radially unbounded if $V(x) \rightarrow \infty$ as $\|x\| \rightarrow \infty$.

\section{GENERALIZED STABILITY THEOREMS FOR NONLINEAR FEEDBACK SYSTEMS}

In this section, we develop generalized Lyapunov and invariant set theorems for nonlinear feedback controlled dynamical systems wherein all regularity assumptions on the Lyapunov function and the closed-loop system dynamics are removed. The following result generalizes the Barbashin-Krasovskii-LaSalle invariant set theorem [27] to the case where the Lyapunov function is lower semicontinuous. For the remainder of the results of this paper, define the notation $\mathcal{R}_{\gamma} \triangleq \bigcap_{c>\gamma} \overline{V^{-1}([\gamma, c])}$, for arbitrary $V: \mathcal{D} \subseteq \mathbb{R}^{n} \rightarrow \mathbb{R}$ and $\gamma \in \mathbb{R}$, and let $\mathcal{M}_{\gamma}$ denote the largest invariant set [with respect to (2)] contained in $\mathcal{R}_{\gamma}$.

Theorem 3.1: Consider the nonlinear feedback controlled dynamical system (2). Let $x(t), t \in \mathcal{I}_{x_{0}}$, denotes the solution to (2), and let $\mathcal{D}_{c} \subset \mathcal{D}$ be a compact positively invariant set with respect to (2). Assume that there exists a lower semicontinuous function $V: \mathcal{D}_{c} \rightarrow \mathbb{R}$ such that $V(x(t)) \leq V(x(\tau)), 0 \leq \tau \leq t$, for all $x_{0} \in \mathcal{D}_{c}$. If $x_{0} \in \mathcal{D}_{c}$, then $x(t) \longrightarrow \mathcal{M} \triangleq \bigcup_{\gamma \in \mathbb{R}} \mathcal{M}_{\gamma}$ as $t \rightarrow \infty$.

Proof: Let $x(t), t \in \mathcal{I}_{x_{0}}$, be the solution to (2) with $x_{0} \in \mathcal{D}_{c}$ so that $[0,+\infty) \subseteq \mathcal{I}_{x_{0}}$. Since $V(\cdot)$ is lower semicontinuous on the compact set $\mathcal{D}_{c}$, there exists $\beta \in \mathbb{R}$ such that $V(x) \geq \beta, x \in \mathcal{D}_{c}$. Hence, since $V(x(t)), t \geq 0$, is nonincreasing, $\gamma_{x_{0}} \triangleq \lim _{t \rightarrow \infty} V(x(t)), x_{0} \in \mathcal{D}_{c}$, exists. Now, for all $p \in \mathcal{P}_{x_{0}}^{+}$there exists an increasing unbounded sequence $\left\{t_{n}\right\}_{n=0}^{\infty}$, with $t_{0}=0$, such that $\lim _{n \rightarrow \infty} x\left(t_{n}\right)=p$. Next, since $V\left(x\left(t_{n}\right)\right), n \geq 0$, is nonincreasing it follows that for all $n \geq 0, \gamma_{x_{0}} \leq V\left(x\left(t_{n}\right)\right) \leq V\left(x\left(t_{N}\right)\right)$, $n \geq N$, or, equivalently, since $\mathcal{D}_{c}$ is positively invariant, $x\left(t_{n}\right) \in V^{-1}\left(\left[\gamma_{x_{0}}, V\left(x\left(t_{N}\right)\right)\right]\right), n \geq N$. Now, since 
$\lim _{n \rightarrow \infty} x\left(t_{n}\right)=p$ it follows that $p \in \overline{V^{-1}\left(\left[\gamma_{x_{0}}, V\left(x\left(t_{n}\right)\right)\right]\right)}$, $n \geq 0$. Furthermore, since $\lim _{n \rightarrow \infty} V\left(x\left(t_{n}\right)\right)=\gamma_{x_{0}}$ it follows that for every $c>\gamma_{x_{0}}$, there exists $n \geq 0$ such that $\gamma_{x_{0}} \leq V\left(x\left(t_{n}\right)\right) \leq c$ which implies that for every $c>\gamma_{x_{0}}$, $p \in \overline{V^{-1}\left(\left[\gamma_{x_{0}}, c\right]\right)}$. Hence, $p \in \mathcal{R}_{\gamma_{x_{0}}}$ which implies that $\mathcal{P}_{x_{0}}^{+} \subseteq \mathcal{R}_{\gamma_{x_{0}}}$. Now, since $\mathcal{D}_{c}$ is compact and positively invariant it follows that the forward solution $x(t), t \geq 0$, to (2) is bounded for all $x_{0} \in \mathcal{D}_{c}$ and hence it follows from Lemma 2.1 that $\mathcal{P}_{x_{0}}^{+}$is a nonempty compact connected invariant set which further implies that $\mathcal{P}_{x_{0}}^{+}$is a subset of the largest invariant set contained in $\mathcal{R}_{\gamma_{x_{0}}}$; that is, $\mathcal{P}_{x_{0}}^{+} \subseteq \mathcal{M}_{\gamma_{x_{0}}}$. Hence, for all $x_{0} \in \mathcal{D}_{c}, \mathcal{P}_{x_{0}}^{+} \subseteq \mathcal{M}$. Finally, since $x(t) \rightarrow \mathcal{P}_{x_{0}}^{+}$as $t \rightarrow \infty$ it follows that $x(t) \rightarrow \mathcal{M}$ as $t \rightarrow \infty$.

Remark 3.1: Note that since $V^{-1}([\gamma, c])=\{x \in$ $\left.\mathcal{D}_{c}: V(x) \geq \gamma\right\} \cap\left\{x \in \mathcal{D}_{c}: V(x) \leq c\right\}$ and $\left\{x \in \mathcal{D}_{c}: V(x) \leq\right.$ $c\}$ is a closed set, it follows that $\hat{\mathcal{R}}_{\gamma, c} \subset\left\{x \in \mathcal{D}_{c}: V(x)<\gamma\right\}$, where $\hat{\mathcal{R}}_{\gamma, c} \triangleq \overline{V^{-1}([\gamma, c])} \backslash V^{-1}([\gamma, c]), c>\gamma$, for a fixed $\gamma \in \mathbb{R}$. Hence,

$$
\mathcal{R}_{\gamma}=\bigcap_{c>\gamma}\left(V^{-1}([\gamma, c]) \cup \hat{\mathcal{R}}_{\gamma, c}\right)=V^{-1}(\gamma) \cup \hat{\mathcal{R}}_{\gamma}
$$

where $\hat{\mathcal{R}}_{\gamma} \triangleq \bigcap_{c>\gamma} \hat{\mathcal{R}}_{\gamma, c}$, is such that $V(x)<\gamma, x \in \hat{\mathcal{R}}_{\gamma}$. Finally, if $V(\cdot)$ is $C^{0}$ then $\hat{\mathcal{R}}_{\gamma, c}=\varnothing, \gamma \in \mathbb{R}, c>\gamma$, and hence $\mathcal{R}_{\gamma}=V^{-1}(\gamma)$.

Remark 3.2: Note that if $V: \mathcal{D}_{c} \rightarrow \mathbb{R}$ is a lower semicontinuous function such that all the conditions of Theorem 3.1 are satisfied, then for every $x_{0} \in \mathcal{D}_{c}$ there exists $\gamma_{x_{0}} \leq V\left(x_{0}\right)$ such that $\mathcal{P}_{x_{0}}^{+} \subseteq \mathcal{M}_{\gamma_{x_{0}}} \subseteq \mathcal{M}$

Remark 3.3: Itisimportant tonote thatasinstandardLyapunov and invariantset theoremsinvolving $C^{1}$ functions, Theorem 3.1 allows one to characterize the invariant set $\mathcal{M}$ without knowledge of the closed-loop system trajectories $x(t), t \in \mathcal{I}_{x_{0}}$. Similar remarks hold for the remainder of the theorems in this section.

Next, we sharpen the results of Theorem 3.1 by providing a refined construction of the invariant set $\mathcal{M}$. In particular, we show that the closed-loop system trajectories converge to a union of largest invariant sets contained on the boundary of the intersections over finite intervals of the closure of generalized Lyapunov level surfaces.

Theorem 3.2: Consider the nonlinear feedback controlled dynamical system (2), let $\mathcal{D}_{c}$ and $\mathcal{D}_{0}$ be compact positively invariant sets with respect to (2) such that $\mathcal{D}_{0} \subset \mathcal{D}_{c} \subset \mathcal{D}$, and let $x(t), t \in \mathcal{I}_{x_{0}}$, denotes the solution to (2) corresponding to $x_{0} \in \mathcal{D}_{c}$. Assume that there exists a lower semicontinuous, positive-definite function $V: \mathcal{D}_{c} \rightarrow \mathbb{R}$ such that $V(x(t)) \leq V(x(\tau)), 0 \leq \tau \leq t$. Furthermore, assume that for all $x_{0} \in \mathcal{D}_{c}, x_{0} \notin \mathcal{D}_{0}$, there exists an increasing unbounded sequence $\left\{t_{n}\right\}_{n=0}^{\infty}$, with $t_{0}=0$, such that

$$
V\left(x\left(t_{n+1}\right)\right)<V\left(x\left(t_{n}\right)\right), \quad n=0,1, \ldots
$$

Then, either $\mathcal{M}_{\gamma} \subset \hat{\mathcal{R}}_{\gamma} \triangleq \mathcal{R}_{\gamma} \backslash V^{-1}(\gamma)$, or $\mathcal{M}_{\gamma}=\varnothing, \gamma>0$. Furthermore, if $x_{0} \in \mathcal{D}_{c}$, then $x(t) \rightarrow \hat{\mathcal{M}} \triangleq \bigcup_{\gamma \in \mathcal{G}} \mathcal{M}_{\gamma}$ as $t \rightarrow$ $\infty$, where $\mathcal{G} \triangleq\left\{\gamma \geq 0: \mathcal{R}_{\gamma} \cap \mathcal{D}_{0} \neq \varnothing\right\}$. If, in addition, $\mathcal{D}_{0} \subset \stackrel{\circ}{\mathcal{D}_{c}}$ and $V(\cdot)$ is continuous on $\mathcal{D}_{0}$, then $\mathcal{D}_{0}$ is locally asymptotically stable and $\mathcal{D}_{c}$ is a subset of the domain of attraction.
Proof: Since $\mathcal{D}_{c}$ is a compact positively invariant set, it follows that for all $x_{0} \in \mathcal{D}_{c}$, the forward solution $x(t), t \geq 0$, to (2) is bounded. Hence, it follows from Lemma 2.1 that, for all $x_{0} \in \mathcal{D}_{c}, \mathcal{P}_{x_{0}}^{+}$is a nonempty, compact, connected invariant set. Next, it follows from Theorem 3.1, Remark 3.2, and the fact that $V(\cdot)$ is positive-definite (with respect to $\mathcal{D}_{c} \backslash \mathcal{D}_{0}$ ), that for every $x_{0} \in \mathcal{D}_{c}$ there exists $\gamma_{x_{0}} \geq 0$ such that $\mathcal{P}_{x_{0}}^{+} \subseteq \mathcal{M}_{\gamma_{x_{0}}} \subseteq \mathcal{R}_{\gamma_{x_{0}}}$. Now, given $x(0) \in V^{-1}\left(\gamma_{x_{0}}\right), \gamma_{x_{0}}>0$, (3) implies that there exists $t_{1}>0$ such that $V\left(x\left(t_{1}\right)\right)<\gamma_{x_{0}}$ and $x\left(t_{1}\right) \notin V^{-1}\left(\gamma_{x_{0}}\right)$. Hence, $V^{-1}\left(\gamma_{x_{0}}\right) \subset \mathcal{R}_{\gamma_{x_{0}}}$ does not contain any invariant set. Alternatively, if $x(0) \in \hat{\mathcal{R}}_{\gamma_{x_{0}}}$ then $V(x(0))<\gamma_{x_{0}}$ and (3) implies that $x(t) \notin V^{-1}\left(\gamma_{x_{0}}\right), t \geq 0$. Hence, any invariant set contained in $\mathcal{R}_{\gamma_{x_{0}}}$ is a subset of $\hat{\mathcal{R}}_{\gamma_{x_{0}}}$, which implies that $\mathcal{M}_{\gamma_{x_{0}}} \subset \hat{\mathcal{R}}_{\gamma_{x_{0}}}, \gamma_{x_{0}}>0$. If $\hat{\gamma}>0$ is such that $\hat{\gamma} \neq \gamma_{x_{0}}$, for all $x_{0} \in \mathcal{D}_{c}$, then there does not exist $x_{0} \in \mathcal{D}_{c}$ such that $\mathcal{P}_{x_{0}}^{+} \subseteq \mathcal{R}_{\hat{\gamma}}$ and hence $\mathcal{M}_{\hat{\gamma}}=\varnothing$. Now, ad absurdum, suppose $\mathcal{D}_{0} \cap \mathcal{P}_{x_{0}}^{+}=$ $\varnothing$. Since $V(\cdot)$ is lower semicontinuous it follows from Theorem 2.1 that there exists $\hat{x} \in \mathcal{P}_{x_{0}}^{+}$such that $\alpha=V(\hat{x}) \leq V(x)$, $x \in \mathcal{P}_{x_{0}}^{+}$. Now, with $x(0)=\hat{x} \notin \mathcal{D}_{0}$ it follows from (3) that there exists an increasing unbounded sequence $\left\{t_{n}\right\}_{n=0}^{\infty}$, with $t_{0}=0$, such that $V\left(x\left(t_{n+1}\right)\right)<V\left(x\left(t_{n}\right)\right), n=0,1, \ldots$, which implies that there exists $t>0$ such that $V(x(t))<\alpha$ which further implies that $x(t) \notin \mathcal{P}_{x_{0}}^{+}$contradicting the fact that $\mathcal{P}_{x_{0}}^{+}$is an invariant set. Hence, there exists $q \in \mathcal{D}_{0}$ such that $q \in \mathcal{P}_{x_{0}}^{+} \subseteq \mathcal{R}_{\gamma_{x_{0}}}$ which implies that $\mathcal{R}_{\gamma_{x_{0}}} \cap \mathcal{D}_{0} \neq \varnothing$. Thus, $\gamma_{x_{0}} \in \mathcal{G}$ for all $x_{0} \in \mathcal{D}_{c}$ which further implies that $\mathcal{P}_{x_{0}}^{+} \subseteq \hat{\mathcal{M}}$. Now, since $x(t) \rightarrow \mathcal{P}_{x_{0}}^{+} \subseteq \hat{\mathcal{M}}$ as $t \rightarrow \infty$ it follows that $x(t) \rightarrow$ $\hat{\mathcal{M}}$ as $t \rightarrow \infty$.

Next, we show that if $V(\cdot)$ is continuous on $\mathcal{D}_{0} \subset \stackrel{\circ}{\mathcal{D}_{c}}$, then the compact positively invariant set $\mathcal{D}_{0}$ of (2) is Lyapunov stable. Let $\mathcal{O}_{1} \subseteq \mathcal{D}_{c}$ be an open neighborhood of $\mathcal{D}_{0}$. Since $\partial \mathcal{O}_{1}$ is compact and $V(x), x \in \mathcal{D}_{c}$, is lower semicontinuous, it follows from Theorem 2.1 that there exists $\alpha=\min _{x \in \partial \mathcal{O}_{1}} V(x)$. Note that $\alpha>0$ since $\mathcal{D}_{0} \cap \partial \mathcal{O}_{1}=\varnothing$ and $V(x)>0, x \in \mathcal{D}_{c}, x \notin \mathcal{D}_{0}$. Next, using the facts that $V(x)=0, x \in \mathcal{D}_{0}$, and $V(\cdot)$ is continuous on $\mathcal{D}_{0}$, it follows that the set $\mathcal{O}_{2} \triangleq\left\{x \in \mathcal{O}_{1}: V(x)<\alpha\right\}^{\circ}$ is not empty. Now, it follows from $V(x(t)) \leq V(x(\tau))$, for all $t \geq \tau \geq 0$ that for all $x(0) \in \mathcal{O}_{2}, V(x(t)) \leq V(x(0))<\alpha, t \geq 0$, which, since $V(x) \geq \alpha, x \in \partial \mathcal{O}_{1}$, implies that $x(t) \notin \partial \mathcal{O}_{1}, t \geq 0$. Hence, for every open neighborhood $\mathcal{O}_{1} \subseteq \mathcal{D}_{c}$ of $\mathcal{D}_{0}$, there exists an open neighborhood $\mathcal{O}_{2} \subseteq \mathcal{O}_{1}$ of $\bar{D}_{0}$ such that, if $x(0) \in \mathcal{O}_{2}$, then $x(t) \in \mathcal{O}_{1}, t \geq 0$, which proves Lyapunov stability of the compact positively invariant set $\mathcal{D}_{0}$ of (2). Finally, from the continuity of $V(\cdot)$ on $\mathcal{D}_{0}$ and the fact that $V(x)=0$ for all $x \in \mathcal{D}_{0}$, it follows that $\mathcal{G}=\{0\}$ and $\hat{\mathcal{M}} \equiv \mathcal{M}_{0}$. Hence, $\mathcal{P}_{x_{0}}^{+} \subseteq \mathcal{D}_{0}$ for all $x_{0} \in \mathcal{D}_{c}$ establishing local asymptotic stability of the compact positively invariant set $\mathcal{D}_{0}$ of (2) with a subset of the domain of attraction given by $\mathcal{D}_{c}$.

Remark 3.4: If in Theorem 3.2 $\hat{\mathcal{M}} \subseteq \mathcal{D}_{0}$, then the compact positively invariant set $\mathcal{D}_{0}$ of (2) is attractive. If, in addition, $V(\cdot)$ is continuous on $\mathcal{D}_{0} \subset \mathcal{D}_{c}$ then the compact positively invariant set $\mathcal{D}_{0}$ of (2) is locally asymptotically stable. In both cases, $\mathcal{D}_{c}$ is a subset of the domain of attraction.

A lower semicontinuous, positive-definite function $V(\cdot)$, with $V(\cdot)$ being continuous on $\mathcal{D}_{0}$, is called a generalized Lyapunov function candidate for the nonlinear feedback controlled 
dynamical system (2). If, additionally, $V(x(t)) \leq V(x(\tau))$, for all $t \geq \tau \geq 0$, where $x(t), t \in \mathcal{I}_{x_{0}}$, denotes the solution to (2) corresponding to $x_{0} \in \mathcal{D}_{c}, V(\cdot)$ is called a generalized Lyapunov function for the nonlinear feedback controlled dynamical system (2). Note that in the case where the function $V(\cdot)$ is $C^{1}$ on $\mathcal{D}_{c}$ in Theorem 3.2, it follows that $V(x(t)) \leq V(x(\tau))$, for all $t \geq \tau \geq 0$, is equivalent to $\dot{V}(x) \triangleq V^{\prime}(x) F(x, \phi(x)) \leq 0$, $x \in \mathcal{D}_{c}$. In this case conditions in Theorem 3.2 specialize to the standard Lyapunov stability conditions [27].

Next, we present a generalized global invariant set theorem for guaranteeing global attraction and global asymptotic stability of a compact positively invariant set of a nonlinear feedback controlled dynamical system.

Theorem 3.3: Consider the nonlinear feedback controlled dynamical system (2) with $\mathcal{D}=\mathbb{R}^{n}$ and $\mathcal{U}=\mathbb{R}^{m}$ and let $x(t)$, $t \in \mathcal{I}_{x_{0}}$, denotes the solution to (2). Assume that there exists a compact positively invariant set $\mathcal{D}_{0}$ with respect to (2) and a lower semicontinuous, radially unbounded, posititive-definite function $V: \mathbb{R}^{n} \rightarrow \mathbb{R}$ such that $V(x(t)) \leq V(x(\tau))$, $0 \leq \tau \leq t$. Then for all $x_{0} \in \mathbb{R}^{n}, x(t) \rightarrow \mathcal{M} \triangleq \bigcup_{\gamma>0} \mathcal{M}_{\gamma}$, as $t \rightarrow \infty$. If, in addition, for all $x_{0} \in \mathbb{R}^{n}, x_{0} \notin \mathcal{D}_{0}$, there exists an increasing unbounded sequence $\left\{t_{n}\right\}_{n=0}^{\infty}$, with $t_{0}=0$, such that $V\left(x\left(t_{n+1}\right)\right)<V\left(x\left(t_{n}\right)\right), n=0,1, \ldots$, then, either $\mathcal{M}_{\gamma} \subset \hat{\mathcal{R}}_{\gamma} \triangleq \mathcal{R}_{\gamma} \backslash V^{-1}(\gamma)$, or $\mathcal{M}_{\gamma}=\varnothing, \gamma>0$. Furthermore, $x(t) \rightarrow \hat{\mathcal{M}} \triangleq \bigcup_{\gamma \in \mathcal{G}} \mathcal{M}_{\gamma}$ as $t \rightarrow \infty$, where $\mathcal{G} \triangleq\left\{\gamma \geq 0: \mathcal{R}_{\gamma} \cap \mathcal{D}_{0} \neq \varnothing\right\}$. Finally, if $V(\cdot)$ is continuous on $\mathcal{D}_{0}$ then the compact positively invariant set $\mathcal{D}_{0}$ of (2) is globally asymptotically stable.

Proof: Note that since $V(x) \rightarrow \infty$ as $\|x\| \rightarrow \infty$ it follows that for every $\beta>0$ there exists $r>0$ such that $V(x)>\beta$ for all $\|x\|>r$, or, equivalently, $V^{-1}([0, \beta]) \subseteq\{x:\|x\| \leq r\}$ which implies that $V^{-1}([0, \beta])$ is bounded for all $\beta>0$. Hence, for all $x_{0} \in \mathbb{R}^{n}, V^{-1}\left(\left[0, \beta_{x_{0}}\right]\right)$ is bounded, where $\beta_{x_{0}} \triangleq V\left(x_{0}\right)$. Furthermore, since $V(\cdot)$ is a positive-definite lower semicontinuous function, it follows that $V^{-1}\left(\left[0, \beta_{x_{0}}\right]\right)$ is closed and, since $V(x(t)), t \geq 0$, is nonincreasing, $V^{-1}\left(\left[0, \beta_{x_{0}}\right]\right)$ is positively invariant. Hence, for every $x_{0} \in \mathbb{R}^{n}, V^{-1}\left(\left[0, \beta_{x_{0}}\right]\right)$ is a compact positively invariant set. Now, with $\mathcal{D}_{c}=V^{-1}\left(\left[0, \beta_{x_{0}}\right]\right)$ it follows from Theorem 3.1 and Remark 3.2 that there exists $\gamma_{x_{0}} \in$ $\left[0, \beta_{x_{0}}\right]$ such that $\mathcal{P}_{x_{0}}^{+} \subseteq \mathcal{M}_{\gamma_{x_{0}}} \subset \hat{\mathcal{R}}_{\gamma_{x_{0}}}$ which implies that $x(t) \rightarrow \mathcal{M}$ as $t \rightarrow \infty$. If, in addition, for all $x_{0} \in \mathbb{R}^{n}, x_{0} \notin \mathcal{D}_{0}$, there exists an increasing unbounded sequence $\left\{t_{n}\right\}_{n=0}^{\infty}$, with $t_{0}=0$, such that $V\left(x\left(t_{n+1}\right)\right)<V\left(x\left(t_{n}\right)\right), n=0,1, \ldots$, holds then it follows from Theorem 3.2 that $x(t) \rightarrow \hat{\mathcal{M}}$ as $t \rightarrow \infty$.

Finally, if $V(\cdot)$ is continuous on $\mathcal{D}_{0}$ then Lyapunov stability follows as in the proof of Theorem 3.2. Furthermore, in this case, $\mathcal{G}=\{0\}$ which implies that $\hat{\mathcal{M}}=\mathcal{M}_{0}$. Hence, $\mathcal{P}_{x_{0}}^{+} \subseteq \mathcal{D}_{0}$ establishing global asymptotic stability of the compact positively invariant set $\mathcal{D}_{0}$ of (2).

Remark 3.5: If in Theorems 3.2 and 3.3, the function $V(\cdot)$ is $C^{1}$ on $\mathcal{D}_{c}$ and $\mathbb{R}^{n}$, respectively, $\mathcal{D}_{0} \equiv\{0\}$, and $V^{\prime}(x) F(x, \phi(x))<0, x \in \mathcal{D}_{c}, x \neq 0$, then every increasing unbounded sequence $\left\{t_{n}\right\}_{n=0}^{\infty}$, with $t_{0}=0$, is such that $V\left(x\left(t_{n+1}\right)\right)<V\left(x\left(t_{n}\right)\right), n=0,1, \ldots$. In this case, Theorems 3.2 and 3.3 specialize to the standard Lyapunov stability theorems for local and global asymptotic stability, respectively, as applied to a closed-loop feedback controlled system.
It is important to note that even though the stability conditions appearing in Theorems 3.1-3.3 are system trajectory dependent, in Section V we present a hierarchical switching nonlinear controller guaranteeing nonlinear system stabilization without requiring knowledge of the closed-loop system trajectories. Finally, we note that the concept of lower semicontinuous Lyapunov functions has been considered in the literature. Specifically, lower semicontinuous Lyapunov functions have been considered in [28] and [30], with [30] focusing on viability theory and differential inclusions. However, the present formulation provides new invariant set stability theorem generalizations characterizing system limit sets in terms of lower semicontinuous Lyapunov functions not considered in [28] or [30].

\section{PARAMETERIZED System EQuilibria AND DOMAINS OF ATTRACTION}

The nonlinear control design framework developed in this paper is predicated on a hierarchical switching nonlinear controller architecture parameterized over a set of system equilibria. It is important to note that both the dynamical system and the controller for each parameterized equilibrium can be nonlinear, and thus, local set point designs are in general nonlinear. Hence, the nonlinear controlled system can be viewed as a collection of controlled subsystems with a hierarchical switching controller architecture. In this section, we concentrate on nonlinear stabilization of the local set points parameterized in $\mathcal{D}$. Specifically, we consider the nonlinear controlled dynamical system (1) with the origin as an equilibrium point corresponding to the control $u=0$, that is, $F(0,0)=0$. Furthermore, we assume that given a mapping $\varphi: \mathcal{D} \times \Lambda \rightarrow \mathcal{U}, \varphi(0,0)=0$, there exists a continuous function $\psi: \Lambda_{o} \rightarrow \mathcal{D}_{o}$, where $\mathcal{D}_{o} \subseteq \mathcal{D}, 0 \in \mathcal{D}_{o}$, and $\Lambda_{0} \subseteq \Lambda, 0 \in \Lambda_{o}$, such that $F\left(x_{\lambda}, \varphi\left(x_{\lambda}, \lambda\right)\right)=0$ with $x_{\lambda}=\psi(\lambda) \in \mathcal{D}_{o}$ for all $\lambda \in \Lambda_{0}$. As discussed in Section II, this is a necessary condition for parametric stability with respect to $\Lambda_{O}$ as defined in [21] and [22], while the implicit function theorem [27, p. 62] provides sufficient conditions for guaranteeing the existence of such a parameterization.

Next, we consider a family of stabilizing feedback control laws given by

$$
\begin{aligned}
\Phi \triangleq & \left\{\phi_{\lambda}: \mathcal{D} \rightarrow \mathcal{U}: \phi_{\lambda} \in C^{0}, \phi_{\lambda}\left(x_{\lambda}\right)=\varphi\left(x_{\lambda}, \lambda\right), \lambda \in \Lambda_{S}\right\} \\
& \Lambda_{S} \subseteq \Lambda_{\circ}
\end{aligned}
$$

such that, for $\phi_{\lambda}(\cdot) \in \Phi, \lambda \in \Lambda_{S}$, the closed-loop nonlinear feedback system

$$
\dot{x}(t)=F\left(x(t), \phi_{\lambda}(x(t))\right), \quad x(0)=x_{0}, \quad t \in \mathcal{I}_{x_{0}}
$$

has an asymptotically stable equilibrium point $x_{\lambda} \in \mathcal{D}_{\circ} \subseteq \mathcal{D}$. Hence, in the terminology of [21] and [22], (5) is parametrically asymptotically stable with respect to $\Lambda_{S} \subseteq \Lambda_{\circ}$. Here, we assume that for each $\lambda \in \Lambda_{S}$, the linear or nonlinear feedback controllers $\phi_{\lambda}(\cdot)$ are given. In particular, these controllers correspond to local set point designs and can be obtained using any appropriate standard linear or nonlinear stabilization scheme with a domain of attraction for each $\lambda \in \Lambda_{S}$. For example, appropriate nonlinear stabilization techniques such as feedback 
linearization, nonlinear $H_{\infty}$ control, constructive nonlinear control, and optimal nonlinear control, as well as linear-quadratic stabilization schemes based on locally approximated linearizations, can be used to design the controllers $\phi_{\lambda}(\cdot)$ for each $\lambda \in$ $\Lambda_{S}$. Furthermore, for an asymptotically stable equilibrium point $x_{\lambda} \in \mathcal{D}_{o} \subseteq \mathcal{D}, \lambda \in \Lambda_{S}$, the domain of attraction $\mathcal{D}_{\lambda}$ of $x_{\lambda}$ is given by

$$
\mathcal{D}_{\lambda} \triangleq\left\{x_{0} \in \mathcal{D} \text { : if } x(0)=x_{0} \text { then } \lim _{t \rightarrow \infty} x(t)=x_{\lambda}\right\} .
$$

Next, given a stabilizing feedback controller $\phi_{\lambda}(\cdot)$ for each $\lambda \epsilon$ $\Lambda_{S}$, we provide a guaranteed subset of the domain of attraction $\mathcal{D}_{\lambda}$ of $x_{\lambda}$ using classical Lyapunov stability theory.

Theorem 4.1 [28]: Let $\lambda \in \Lambda_{S}$. Consider the closed-loop nonlinear system (5) with $\phi_{\lambda}(\cdot) \in \Phi$ and let $x_{\lambda} \in \mathcal{D}_{o} \subseteq \mathcal{D}$ be an equilibrium point of (5). Furthermore, let $\mathcal{X}_{\lambda} \subset \mathcal{D}$ be a compact neighborhood of $x_{\lambda}$. Then $x_{\lambda} \in \mathcal{D}_{o} \subseteq \mathcal{D}$ is locally asymptotically stable if and only if there exists a $C^{1}$ function $V_{\lambda}: \mathcal{X}_{\lambda} \rightarrow \mathbb{R}$ such that $V_{\lambda}\left(x_{\lambda}\right)=0, V_{\lambda}(x)>0, x \in \mathcal{X}_{\lambda} \backslash$ $\left\{x_{\lambda}\right\}$, and $\dot{V}_{\lambda}(x) \triangleq V_{\lambda}^{\prime}(x) F\left(x, \phi_{\lambda}(x)\right)<0, x \in \mathcal{X}_{\lambda} \backslash\left\{x_{\lambda}\right\}$. In addition, a subset of the domain of attraction of $x_{\lambda}$ is given by $\mathcal{D}_{\lambda} \triangleq V_{\lambda}^{-1}\left(\left[0, c_{\lambda}\right]\right)$, where $c_{\lambda} \triangleq \max \left\{\beta>0: V_{\lambda}^{-1}([0, \beta]) \subseteq\right.$ $\left.\mathcal{X}_{\lambda}\right\}$.

Remark 4.1: It follows from Theorem 4.1 that for all $x_{0} \in$ $\mathcal{D}_{\lambda}, \lim _{t \rightarrow \infty} V_{\lambda}(x(t))=0$ or, equivalently, for each $\delta>0$ there exists a finite time $T>0$ such that $V_{\lambda}(x(t)) \leq \delta, t \geq T$. Hence, given the initial condition $x_{0} \in \mathcal{D}_{\lambda}$, it follows that for every $\delta>0$ there exists a finite time $T>0$ such that $x(t) \in$ $V_{\lambda}^{-1}([0, \delta]), t \geq T$.

We stress that the aim of Theorem 4.1 is not to make direct comparisons with existing methods for estimating domains of attraction, but rather in aiding to provide a streamlined presentation of the main results of Section $\mathrm{V}$ requiring estimates of domains of attraction for local set point designs.

\section{NONLINEAR SYSTEM STABILIZATION VIA A HiERARCHICAL SWITCHING CONTROLLER ARCHITECTURE}

In this section, we develop a nonlinear stabilization framework predicated on a hierarchical switching controller architecture parameterized over a set of moving system equilibria. Specifically, using equilibria-dependent Lyapunov functions, or instantaneous (with respect to a given parameterized equilibrium manifold) Lyapunov functions, a hierarchical nonlinear control strategy is developed that stabilizes a given nonlinear system by stabilizing a collection of nonlinear controlled subsystems while providing an explicit expression for a guaranteed domain of attraction. A switching nonlinear controller architecture is developed based on a generalized lower semicontinuous Lyapunov function obtained by minimizing a potential function, associated with each domain of attraction, over a given switching set induced by the parameterized system equilibria. In the case where one of the parameterized equilibrium points is globally asymptotically stable with a given subcontroller and a structural topological constraint is enforced on the switching set, the proposed nonlinear stabilization framework guarantees global asymptotic stability of any given system equilibrium on the parameterized equilibrium manifold.
To state the main results of this section several definitions and a key assumption are needed. Recall that the set $\Lambda_{S} \subseteq \Lambda_{o}$, $0 \in \Lambda_{S}$, is such that for every $\lambda \in \Lambda_{S}$ there exists a feedback control law $\phi_{\lambda}(\cdot) \in \Phi$ such that the equilibrium point $x_{\lambda} \in$ $\mathcal{D}_{o} \subseteq \mathcal{D}$ of (5) is asymptotically stable with an estimate of the domain of attraction given by $\mathcal{D}_{\lambda}$. Since $x_{\lambda}, \lambda \in \Lambda_{S}$, is an asymptotically stable equilibrium point of (5), it follows that the assumptions of Theorem 4.1 are satisfied, and hence, without loss of generality, we can take $\mathcal{D}_{\lambda}, \lambda \in \Lambda_{S}$, given by Theorem 4.1. Furthermore, we assume that the set-valued map $\Psi: \Lambda_{S} \leadsto$ $2^{\mathcal{D}}$, where $2^{\mathcal{D}}$ denotes the collection of all subsets of $\mathcal{D}$, is such that $\mathcal{D}_{\lambda}=\Psi(\lambda), \lambda \in \Lambda_{S}$, is continuous. Here, continuity of a set-valued map is defined in the sense of [30, p. 56] and has the property that the limit of a sequence of a continuous set-valued map is the value of the map at the limit of the sequence. In particular, since $\mathcal{D}_{\lambda}, \lambda \in \Lambda_{S}$, is given by Theorem 4.1, the continuity of the set-valued map $\Psi(\cdot)$ is guaranteed provided that $V_{\lambda}(x), x \in \mathcal{D}_{\lambda}$, and $c_{\lambda}$ are continuous functions of the parameter $\lambda \in \Lambda_{S}$. Next, let $\mathcal{S} \subseteq \Lambda_{S}, 0 \in \mathcal{S}$, denote a switching set such that the following key assumption is satisfied.

Assumption 5.1: The switching set $\mathcal{S} \subseteq \Lambda_{S}$ is such that the following two properties hold.

1) There exists a continuous positive-definite function $p: \mathcal{S} \rightarrow \mathbb{R}$ such that for all $\lambda \in \mathcal{S}, \lambda \neq 0$, there exists $\lambda_{1} \in \mathcal{S}$ such that $p\left(\lambda_{1}\right)<p(\lambda), x_{\lambda} \in \mathcal{D}_{\lambda_{1}}$.

2) If $\lambda, \lambda_{1} \in \mathcal{S}, \lambda \neq \lambda_{1}$, is such that $p(\lambda)=p\left(\lambda_{1}\right)$, then $\mathcal{D}_{\lambda} \cap \mathcal{D}_{\lambda_{1}}=\varnothing$.

Note that Assumption 5.1 assumes the existence of a positive-definite potential function $p(\lambda)$ for all $\lambda$ in the switching set $\mathcal{S}$. It follows that, for each $\lambda \in \mathcal{S}$, there exists an equilibrium point $x_{\lambda}$ with an associated domain of attraction $\mathcal{D}_{\lambda}$, and potential value $p(\lambda)$. Hence, every domain of attraction has an associated value of the potential function such that, according to 2), domains of attraction corresponding to different local set point designs intersect each other only if their corresponding potentials are different. In particular, given $\mathcal{D}_{\lambda}, \lambda \in \mathcal{S} \backslash\{0\}$, it is always possible to find at least one intersecting domain of attraction $\mathcal{D}_{\lambda_{1}}, \lambda_{1} \in \mathcal{S}$, such that the potential function decreases and $\mathcal{D}_{\lambda_{1}}$ contains $x_{\lambda}$ as an internal point. This guarantees that if a forward trajectory $x(t), t \geq 0$, of the controlled system approaches $x_{\lambda}$, then there exists a finite time $T>0$ such that the trajectory enters $\mathcal{D}_{\lambda_{1}}$. Finally, it is important to note that the switching set $\mathcal{S}$ is arbitrary. In particular, we do not assume that $\mathcal{S}$ is countable or countably infinite. For example, the switching set $\mathcal{S}$ can have a hybrid topological structure involving isolated points and closed sets homeomorphic to intervals on the real line.

Next, we show that Assumption 5.1 implies that every level set of the potential function $p(\cdot)$ is either empty or consists of only isolated points. Furthermore, in a neighborhood of the origin every level set of $p(\cdot)$ consists of at most one isolated point. For the statement of this result, let $\mathcal{B}_{\lambda}(r), \lambda \in \mathcal{U}_{o}, r>$ 0 , denote the open ball centered at $x_{\lambda}$ with radius $r$, that is, $\mathcal{B}_{\lambda}(r) \triangleq\left\{x \in \mathcal{D}:\left\|x-x_{\lambda}\right\|<r\right\}$.

Proposition 5.1: Let $\mathcal{S} \subseteq \Lambda_{S}$ be such that Assumption 5.1 holds. Then for every $\alpha>0, p^{-1}(\alpha)$ is either empty or consists of only isolated points. Furthermore, there exists $\beta>0$ such 
that for every $\alpha<\beta, p^{-1}(\alpha)$ consists of at most one isolated point.

Proof: Suppose, ad absurdum, that there exists $\hat{\lambda} \in p^{-1}(\alpha), \alpha>0$, such that $\hat{\lambda}$ is not an isolated point in $p^{-1}(\alpha)$. Now, let $\hat{\mathcal{N}} \subset p^{-1}(\alpha)$ be a neighborhood of $\hat{\lambda}$ and note that, by continuity of $\psi(\cdot)$ and the fact that $\hat{\lambda} \in p^{-1}(\alpha)$ is not an isolated point, for every $\epsilon>0$, there exist $\lambda_{1}, \lambda_{2} \in \hat{\mathcal{N}}$ such that $\left\|x_{\lambda_{1}}-x_{\lambda_{2}}\right\|<\epsilon$, and $p\left(\lambda_{1}\right)=p\left(\lambda_{2}\right)=\alpha$. However, since $x_{\lambda_{1}} \in \stackrel{\circ}{\mathcal{D}}_{\lambda_{1}}$, it follows that there exists $r>0$ such that $\mathcal{B}_{\lambda_{1}}(r) \subseteq \mathcal{D}_{\lambda_{1}}$. Now, choosing $\epsilon<r$ yields $x_{\lambda_{2}} \in \mathcal{B}_{\lambda_{1}}(r) \subseteq \mathcal{D}_{\lambda_{1}}$ and $x_{\lambda_{2}} \in \mathcal{D}_{\lambda_{2}}$ which implies that $\mathcal{D}_{\lambda_{1}} \cap \mathcal{D}_{\lambda_{2}} \neq \varnothing$ contradicting ii) of Assumption 5.1. Hence, if $p^{-1}(\alpha), \alpha>0$, is nonempty, it must consist of only isolated points.

Next, suppose, ad absurdum, that for all $\delta>0$ there exist two isolated points $\lambda_{1}, \lambda_{2} \in \hat{\mathcal{N}_{\delta}} \triangleq\{\lambda \in \mathcal{S}:\|\lambda\|<\delta\}$ such that $p\left(\lambda_{1}\right)=p\left(\lambda_{2}\right)$. Now, repeating the above arguments leads to a contradiction. Hence, there exists $\hat{\delta}>0$ such that if $\lambda_{1} \in \hat{\mathcal{N}}_{\hat{\delta}}$, then $\hat{\mathcal{N}}_{\hat{\delta}} \cap p^{-1}\left(p\left(\lambda_{1}\right)\right)=\left\{\lambda_{1}\right\}$. Now, since $p(\cdot)$ is continuous and positive definite, it follows that there exists $\beta>0$ such that $p^{-1}(\alpha) \subseteq \hat{\mathcal{N}}_{\hat{\delta}}, \alpha<\beta$, and hence $p^{-1}(\alpha), \alpha<\beta$, consists of at most one isolated point.

Note that Proposition 5.1 implies that, if $p^{-1}(\alpha), \alpha>0$, is bounded, then there exists a finite distance between isolated points contained in $p^{-1}(\alpha)$ which consists of at most a finite number of isolated points. Finally, since in a neighborhood of the origin every level set of $p(\cdot)$ consists of at most one isolated point, a particular topology for $\mathcal{S}$, in a neighborhood of the origin, is homeomorphic to the interval $[0, a], a>0$, with $0 \in \mathcal{S}$ corresponding to $0 \in \mathbb{R}$.

Now, for every $x \in \mathcal{D}_{c} \triangleq \bigcup_{\lambda \in \mathcal{S}} \mathcal{D}_{\lambda}$, define the viable switching set $\mathcal{V}_{\mathcal{S}}(x) \triangleq\left\{\lambda \in \mathcal{S}: x \in \mathcal{D}_{\lambda}\right\}$, which contains all $\lambda \in \mathcal{S}$ such that $x \in \mathcal{D}_{\lambda}$. Note that if we consider a sequence $\left\{\lambda_{n}\right\}_{n=1}^{\infty} \subset \mathcal{V}_{\mathcal{S}}(x)$, that is, $x \in \mathcal{D}_{\lambda_{n}}$, such that $\lim _{n \rightarrow \infty} \lambda_{n}=\bar{\lambda}$, it follows from the continuity of the set-valued map $\Psi(\cdot)$ that $x \in \mathcal{D}_{\bar{\lambda}}$. Thus, $\bar{\lambda} \in \mathcal{V}_{\mathcal{S}}(x)$ which implies that $\mathcal{V}_{\mathcal{S}}(x)$ is a nonempty closed set since it contains all of its accumulation points.

Next, we introduce the switching function $\lambda_{\mathcal{S}}(x), x \in \mathcal{D}_{c}$, such that the following definitions hold

$$
\begin{gathered}
V(x) \triangleq p\left(\lambda_{\mathcal{S}}(x)\right), \quad \lambda_{\mathcal{S}}(x) \triangleq \arg \min \left\{p(\lambda): \lambda \in \mathcal{V}_{\mathcal{S}}(x)\right\} \\
x \in \mathcal{D}_{c} .
\end{gathered}
$$

In particular, $\lambda_{\mathcal{S}}(x), x \in \mathcal{D}_{c}$, corresponds to the value at which $p(\lambda)$ is minimized wherein $\lambda$ belongs to the viable switching set. The following proposition shows that "min" in (7) is attained and hence $V(x)$ is well defined.

Proposition 5.2: Let $\mathcal{S} \subseteq \Lambda_{S}$ and let $p: \mathcal{S} \rightarrow \mathbb{R}$ be a continuous positive-definite function such that Assumption 5.1 holds. Then, for all $x \in \mathcal{D}_{c}$, there exists a unique $\lambda_{\mathcal{S}}(x) \in \mathcal{V}_{\mathcal{S}}(x)$ such that $p\left(\lambda_{\mathcal{S}}(x)\right)=\min \left\{p(\lambda): \lambda \in \mathcal{V}_{\mathcal{S}}(x)\right\}$.

Proof: Existence follows from the fact that $p(\cdot)$ is lower bounded and $\mathcal{V}_{\mathcal{S}}(x), x \in \mathcal{D}_{c}$, is a nonempty closed set. Now, to prove uniqueness, suppose ad absurdum $\lambda_{\mathcal{S}}(x)$ is not unique. In this case, there exist $\lambda_{1}, \lambda_{2} \in \mathcal{S}, \lambda_{1} \neq \lambda_{2}$, such that $p\left(\lambda_{1}\right)=$ $p\left(\lambda_{2}\right)$ and $x \in \mathcal{D}_{\lambda_{1}} \cap \mathcal{D}_{\lambda_{2}} \neq \varnothing$ which contradicts ii) in Assumption 5.1.

The next result shows that $V(\cdot)$ given by (7) is a generalized Lyapunov function candidate, i.e., $V(\cdot)$ is lower semicontinuous on $\mathcal{D}_{c}$.

Theorem 5.1: Let $\mathcal{S} \subseteq \Lambda_{S}$ be such that Assumption 5.1 holds. Then the function $\bar{V}(x)=p\left(\lambda_{\mathcal{S}}(x)\right), x \in \mathcal{D}_{c}$, is lower

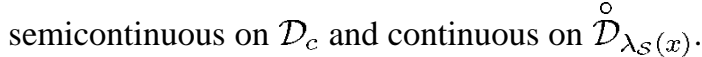

Proof: Let the sequence $\left\{x_{n}\right\}_{n=0}^{\infty} \subset \mathcal{D}_{c}$ be such that $\lim _{n \rightarrow \infty} x_{n}=\hat{x}$ and define $\hat{\lambda} \triangleq \liminf _{n \rightarrow \infty} \lambda_{\mathcal{S}}\left(x_{n}\right)$. Here we assume without loss of generality that $\left\{\lambda_{\mathcal{S}}\left(x_{n}\right)\right\}_{n=0}^{\infty}$ converges to $\hat{\lambda}$; if this is not the case, it is always possible to construct a subsequence having this property. Since $p(\cdot)$ is continuous [and hence $p\left(\lim _{n \rightarrow \infty} \lambda_{\mathcal{S}}\left(x_{n}\right)\right)=\lim _{n \rightarrow \infty} p\left(\lambda_{\mathcal{S}}\left(x_{n}\right)\right)$ ], it suffices to show that $V(\hat{x}) \leq p(\hat{\lambda})$. Suppose, ad absurdum, that $V(\hat{x})>p(\hat{\lambda})$. In this case, there exists a positive integer $n_{0}$ such that $V(\hat{x})>V\left(x_{n}\right), n \geq n_{0}$. Now, since by definition $\lambda_{\mathcal{S}}(\hat{x})$ minimizes $p(\lambda)$ for $\lambda \in \overline{\mathcal{V}}_{\mathcal{S}}(\hat{x})$, it follows that $V(\hat{x}) \leq$ $p(\lambda), \lambda \in \mathcal{V}_{\mathcal{S}}(\hat{x})$. Hence, since $V(\hat{x})>V\left(x_{n}\right)=p\left(\lambda_{\mathcal{S}}\left(x_{n}\right)\right)$, $n \geq n_{0}$, it follows that $\lambda_{\mathcal{S}}\left(x_{n}\right) \notin \mathcal{V}_{\mathcal{S}}(\hat{x})$ and $\hat{x} \notin \mathcal{D}_{\lambda_{\mathcal{S}}\left(x_{n}\right)}$, $n \geq n_{0}$. Now, define the closed set $\hat{\mathcal{D}} \triangleq \bigcup_{n=n_{0}}^{\infty} \mathcal{D}_{\lambda_{\mathcal{S}}\left(x_{n}\right)}$ such that $\left\{x_{n}\right\}_{n=n_{0}}^{\infty} \subset \hat{\mathcal{D}}$. Since $\hat{\mathcal{D}}$ is closed, it follows that $\hat{x} \in \hat{\mathcal{D}}$ which implies that there exist $n_{1} \geq n_{0}$ such that $\hat{x} \in \mathcal{D}_{\lambda_{\mathcal{S}}\left(x_{n_{1}}\right)}$ which is a contradiction.

To show that $V(x)$ is continuous on ${\stackrel{\circ}{\lambda_{\mathcal{S}}(\hat{x})}}$ it need only be shown that $V(\hat{x})$ is upper semicontinuous on ${\stackrel{\mathcal{D}}{\lambda_{\mathcal{S}}(\hat{x})}}_{\text {, or, equiv- }}$ alently, $V(\hat{x}) \geq p(\hat{\lambda})$. Since $\lim _{n \rightarrow \infty} x_{n}=\hat{x}$ and $\hat{x} \in \stackrel{\circ}{\mathcal{D}}_{\lambda_{\mathcal{S}}(\hat{x})}$, there exists a positive integer $n_{2}$ such that $x_{n} \in \mathcal{D}_{\lambda_{\mathcal{S}}(\hat{x})}, n \geq$ $n_{2}$. Hence, $\lambda_{\mathcal{S}}(\hat{x}) \in \mathcal{V}_{\mathcal{S}}\left(x_{n}\right)$ and $V(\hat{x}) \geq V\left(x_{n}\right), n \geq n_{2}$, which implies that $V(\hat{x}) \geq p(\hat{\lambda})$.

Next, we show that with the hierarchical nonlinear feedback control strategy $u=\phi_{\lambda_{\mathcal{S}}(x)}(x), x \in \mathcal{D}_{c}, V(\cdot)$ given by (7) is a generalized Lyapunov function for the nonlinear feedback controlled dynamical system (2). The controller notation $\phi_{\lambda_{\mathcal{S}}(x)}(x)$ denotes a switching nonlinear feedback controller where the switching function $\lambda_{\mathcal{S}}(x), x \in \mathcal{D}_{c}$, is such that definition (7) of the generalized Lyapunov function $V(x), x \in \mathcal{D}_{c}$, holds for a given potential function $p(\cdot)$ and switching set $\mathcal{S}$ satisfying Assumption 5.1. Furthermore, note that since $\phi_{\lambda_{\mathcal{S}}(x)}(x)$ is defined for $x \in \mathcal{D}_{c}$, it follows that the solution $x(\cdot)$ to (2) with $x_{0} \in \mathcal{D}_{c}$ and $u=\phi_{\lambda_{\mathcal{S}}(x)}(x)$ is defined for all values of $t \in \mathcal{I}_{x_{0}}$ such that $x(t) \in \mathcal{D}_{c}$. However, as will be shown, since $\mathcal{D}_{c}$ is a positively invariant set, $[0,+\infty) \subseteq \mathcal{I}_{x_{0}}$, while if $x_{0} \in \mathcal{D}_{c}$ is such that $x(t), t<0$, is always contained in $\mathcal{D}_{c}$, then $\mathcal{I}_{x_{0}}=\mathbb{R}$. Finally, note that since the solution $x(t), t \in \mathcal{I}_{x_{0}}$, to (2) with $x_{0} \in \mathcal{D}_{c}$ and $u=\phi_{\lambda_{\mathcal{S}}(x)}(x)$ is continuous, it follows from Theorem 5.1 that $V(x(t)), t \in \mathcal{I}_{x_{0}}$, is right continuous. Hence, using the continuity of $p(\cdot)$ and the definition of $V(x)$, $x \in \mathcal{D}_{c}$, it follows that $\lambda_{\mathcal{S}}(x(t)), t \in \mathcal{I}_{x_{0}}$, is also right continuous. Now, the continuity of $F(\cdot, \cdot)$ and $\phi_{\lambda}(\cdot), \lambda \in \Lambda_{S}$, imply that $F\left(x(t), \phi_{\lambda_{\mathcal{S}}(x(t))}(x(t))\right), t \in \mathcal{I}_{x_{0}}$, is right continuous.

Theorem 5.2: Consider the nonlinear controlled dynamical system (1) with $F(0,0)=0$ and assume there exists a continuous function $\psi: \Lambda_{o} \rightarrow \mathcal{D}_{o}, 0 \in \Lambda_{o}$, parameterizing an equilibrium manifold of (1), such that $x_{\lambda}=\psi(\lambda), \lambda \in \Lambda_{\circ}$. Furthermore, assume that there exists a $C^{0}$ feedback control law $\phi_{\lambda}(\cdot)$, 
$\lambda \in \Lambda_{S} \subseteq \Lambda_{o}$ with $0 \in \Lambda_{S}$, that locally stabilizes $x_{\lambda}$ with a domain of attraction estimate $\mathcal{D}_{\lambda}$ and let $\mathcal{S} \subseteq \Lambda_{S}, 0 \in \mathcal{S}$, be such that Assumption 5.1 holds. If $\lambda_{\mathcal{S}}(x), x \in \mathcal{D}_{c}$, is such that $V(x), x \in \mathcal{D}_{c}$, given by (7) holds and $x(t), t \in \mathcal{I}_{x_{0}}$, is the solution to (1) with $x(0)=x_{0} \in \mathcal{D}_{c}$ and feedback control law $u=\phi_{\lambda_{\mathcal{S}}(x)}(x), x \in \mathcal{D}_{c}$, then $\mathcal{D}_{c}$ is positively invariant and $V(x(t)), t \in \mathcal{I}_{x_{0}}$, is nonincreasing. Furthermore, for all $t_{1}, t_{2} \in \mathcal{I}_{x_{0}}, V(x(t))=V\left(x\left(t_{1}\right)\right), t \in\left[t_{1}, t_{2}\right]$, if and only if $\lambda_{\mathcal{S}}(x(t))=\lambda_{\mathcal{S}}\left(x\left(t_{1}\right)\right), t \in\left[t_{1}, t_{2}\right]$. Finally, for all $t \in \mathcal{I}_{x_{0}}$ such that $\lambda_{\mathcal{S}}(x(t)) \neq 0$, there exists a finite time $T>0$ such that $V(x(t+T))<V(x(t))$.

Proof: First, note that $x \in \partial \mathcal{D}_{c}$ implies $x \in \partial \mathcal{D}_{\lambda_{\mathcal{S}}(x)}$. Since $\phi_{\lambda_{\mathcal{S}}(x)}$ stabilizes $x_{\lambda_{\mathcal{S}}(x)}$ with domain of attraction $\mathcal{D}_{\lambda_{\mathcal{S}}(x)}$, it follows that, for all $x \in \partial \mathcal{D}_{c}$, the flow of $F\left(x, \phi_{\lambda_{\mathcal{S}}(x)}\right)$ is directed toward the interior of $\mathcal{D}_{\lambda_{\mathcal{S}}(x)}$ and consequently toward the interior of $\mathcal{D}_{c}$, which proves positive invariance of $\mathcal{D}_{c}$. Next, let $x(t), t \in \mathcal{I}_{x_{0}}$, satisfy (1) with $u(t)=\phi_{\lambda_{t}}(x(t))$, where $\lambda_{t} \triangleq \lambda_{\mathcal{S}}(x(t))$, and let, for an arbitrary time $t_{1} \in \mathcal{I}_{x_{0}}$, the feedback control law $u=\phi_{\lambda_{t_{1}}}(x)$ asymptotically stabilize the equilibrium point $x_{\lambda_{t_{1}}}$ of (1) with domain of attraction $\mathcal{D}_{\lambda_{t_{1}}}$. Since $x\left(t_{1}\right) \in \mathcal{D}_{\lambda_{t_{1}}}$, it follows from Theorem 4.1 that there exists a $C^{1}$ Lyapunov function $V_{\lambda_{t_{1}}}(\cdot)$ such that $\dot{V}_{\lambda_{t_{1}}}(x(t)) \triangleq V_{\lambda_{t_{1}}}^{\prime}(x(t)) F\left(x(t), \phi_{\lambda_{\mathcal{S}}(x(t))}(x(t))\right)$, $t \in \mathcal{I}_{x_{0}}$, and $\dot{V}_{\lambda_{t_{1}}}\left(x\left(t_{1}\right)\right)=V_{\lambda_{t_{1}}}^{\prime}\left(x\left(t_{1}\right)\right) F_{\lambda_{t_{1}}}\left(x\left(t_{1}\right)\right)<0$. Next, since $F\left(x(t), \phi_{\lambda_{S}(x(t))}(x(t))\right), t \in \mathcal{I}_{x_{0}}$, is right continuous, it follows that there exists $\delta>0$ such that $\dot{V}_{\lambda_{t_{1}}}(x(t))<0$, $t \in\left[t_{1}, t_{1}+\delta\right]$, which implies that $V_{\lambda_{t_{1}}}(x(t))<V_{\lambda_{t_{1}}}\left(x\left(t_{1}\right)\right)$, $t \in\left[t_{1}, t_{1}+\delta\right]$. Hence, $x(t) \in \mathcal{D}_{\lambda_{t}}, t \in\left[t_{1}, t_{1}+\delta\right]$, and $\lambda_{t_{1}} \in \mathcal{V}_{\mathcal{S}}(x(t)), t \in\left[t_{1}, t_{1}+\delta\right]$, which implies that $V(x(t)) \leq V\left(x\left(t_{1}\right)\right)=p\left(\lambda_{t_{1}}\right), t \in\left[t_{1}, t_{1}+\delta\right]$. Now, since $t_{1} \in \mathcal{I}_{x_{0}}$ is arbitrary, it follows that $V(x(t)), t \in \mathcal{I}_{x_{0}}$, is a nonincreasing function along the trajectories $x(t), t \in \mathcal{I}_{x_{0}}$, of (1) with $u(t)=\phi_{\lambda_{t}}(x(t))$.

Next, assume that $x(t) \in \mathcal{D}_{\lambda_{t_{1}}}$ and $V(x(t))=V\left(x\left(t_{1}\right)\right), t \in$ $\left[t_{1}, t_{1}+\hat{\delta}\right]$. Now, suppose, ad absurdum, that $\lambda_{t}, t \in\left[t_{1}, t_{1}+\hat{\delta}\right]$, is not constant, that is, there exists $t_{2} \in\left(t_{1}, t_{1}+\hat{\delta}\right]$ such that $\lambda_{t_{1}} \neq \lambda_{t_{2}}$. In this case $x\left(t_{2}\right) \in \mathcal{D}_{\lambda_{t_{1}}} \cap \mathcal{D}_{\lambda_{t_{2}}}$ and $p\left(\lambda_{t_{1}}\right)=$ $p\left(\lambda_{t_{2}}\right)$, which contradicts ii) of Assumption 5.1. Hence, it follows that if $V(x(t))=V\left(x\left(t_{2}\right)\right), t \in\left[t_{1}, t_{1}+\hat{\delta}\right]$, then $\lambda_{t}=\lambda_{t_{1}}$, $t \in\left[t_{1}, t_{1}+\hat{\delta}\right]$. Conversely, if for $t_{1}, t_{2} \in \mathcal{I}_{x_{0}}, \lambda_{t}=\lambda_{t_{1}}$, $t \in\left[t_{1}, t_{2}\right]$, then $V(x(t))=V\left(x\left(t_{1}\right)\right), t \in\left[t_{1}, t_{2}\right]$, is immediate.

Finally, for an arbitrary $t_{1} \in \mathcal{I}_{x_{0}}$, suppose, ad absurdum, that $V(x(t))=V\left(x\left(t_{1}\right)\right) \neq 0, t \geq t_{1}$, or, equivalently, $\lambda_{t}=\lambda_{t_{1}} \in$ $\mathcal{S} \backslash\{0\}, t \geq t_{1}$. Then the feedback control law $\phi_{\lambda_{t}}(\cdot)=\phi_{\lambda_{t_{1}}}(\cdot)$ stabilizes the equilibrium point $x_{\lambda_{t_{1}}}$. In this case, it follows from Assumption 5.1 that there exists $\lambda_{1} \neq \lambda_{t_{1}}$ such that $p\left(\lambda_{1}\right)<$ $V\left(x\left(t_{1}\right)\right)$ and $x_{\lambda_{t_{1}}} \in \stackrel{\circ}{\mathcal{D}}_{\lambda_{1}}$, which implies that there exists $\alpha>0$ such that $x_{\lambda_{t_{1}}} \in V_{\lambda_{t_{1}}}^{-1}([0, \alpha]) \subseteq \mathcal{D}_{\lambda_{1}}$. Hence, it follows from Remark 4.1 that $x(t)$ approaches the level set $V_{\lambda_{t_{1}}}^{-1}(\alpha)$ in a finite time $T>0$ so that $V\left(x\left(t_{1}+T\right)\right) \leq p\left(\lambda_{1}\right)<V\left(x\left(t_{1}\right)\right)$, which contradicts the original supposition.

Next, we show that the hierarchical switching nonlinear controller $u=\phi_{\lambda_{\mathcal{S}}(x)}(x), x \in \mathcal{D}_{c}$, guarantees that the generalized Lyapunov function (7) is nonincreasing along the closed-loop system trajectories with strictly decreasing values only at the switching times which occur when the closed-loop system trajectory enters a new domain of attraction with an associated lower potential value.

Corollary 5.1: Consider the nonlinear controlled dynamical system (1) with $F(0,0)=0$ and assume the hypothesis of Theorem 5.2 holds. Then $V(x(t)), t \geq 0$, is strictly decreasing only at the switching times which occur when the trajectory $x(t)$, $t \in \mathcal{I}_{x_{0}}$, enters a new domain of attraction with an associated lower potential value.

Proof: First, we consider the case where $x\left(t_{2}\right) \in \stackrel{\circ}{\mathcal{D}}_{\lambda_{t_{2}}}$, with $\lambda_{t_{2}} \triangleq \lambda_{\mathcal{S}}\left(x\left(t_{2}\right)\right)$ and $t_{2}>0$. It follows from the continuity of the closed-loop system trajectories $x(\cdot)$ that there exists $t_{1}<$ $t_{2}$ such that $x\left(t_{1}\right) \in \mathcal{D}_{\lambda_{t_{2}}}$, which implies that $\lambda_{t_{2}} \in \mathcal{V}_{\mathcal{S}}\left(x\left(t_{1}\right)\right)$ and $V\left(x\left(t_{1}\right)\right) \leq V\left(x\left(t_{2}\right)\right)$. Since $V(x(t)), t \geq 0$, is a nonincreasing function of time, it follows that $V(x(t))=V\left(x\left(t_{2}\right)\right)$, $t \in\left[t_{1}, t_{2}\right]$. Alternatively, assume that $x\left(t_{2}\right) \in \partial \mathcal{D}_{\lambda_{t_{2}}}$, and suppose, ad absurdum, that there exists $t_{1}<t_{2}$ such that $x\left(t_{1}\right) \in$ $\mathcal{D}_{\lambda_{t_{2}}}$. Then $\lambda_{t}=\lambda_{t_{2}}, t \in\left[t_{1}, t_{2}\right]$, and, since $V_{\lambda_{t_{2}}}(x), x \in$ $\mathcal{D}_{\lambda_{t_{2}}}$, attains its maximum at $x\left(t_{2}\right) \in \partial \mathcal{D}_{\lambda_{t_{2}}}$, it follows that $V_{\lambda_{t_{2}}}(x(t)) \leq V_{\lambda_{t_{2}}}\left(x\left(t_{2}\right)\right), t \in\left[t_{1}, t_{2}\right]$, which contradicts the fact that $V_{\lambda_{t_{2}}}(x(t)), t \geq 0$, is a decreasing function of time. Hence, $x(t) \notin \mathcal{D}_{\lambda_{t_{2}}}$ and $V(x(t))<V\left(x\left(t_{2}\right)\right)$, for all $t<$ $t_{2}$.

Finally, we present the main result of this section. Specifically, we show that the hierarchical switching nonlinear controller given by $u=\phi_{\lambda_{\mathcal{S}}(x)}(x), x \in \mathcal{D}_{c}$, guarantees that the closed-loop system trajectories converge to a union of largest invariant sets contained on the boundary of intersections over finite intervals of the closure of the generalized Lyapunov level surfaces. In addition, if the switching set $\mathcal{S}$ is homeomorphic to an interval on the real line and/or consists of only isolated points, then the hierarchical switching nonlinear controller establishes asymptotic stability of the origin.

Theorem 5.3: Consider the nonlinear controlled dynamical system (1) with $F(0,0)=0$ and assume there exists a continuous function $\psi: \Lambda_{O} \rightarrow \mathcal{D}_{O}, 0 \in \Lambda_{O}$, parameterizing an equilibrium manifold of (1), such that $x_{\lambda}=\psi(\lambda), \lambda \in \Lambda_{0}$. Furthermore, assume that there exists a $C^{0}$ feedback control law $\phi_{\lambda}(\cdot)$, $\lambda \in \Lambda_{S} \subseteq \Lambda_{o}$ with $0 \in \Lambda_{S}$, that locally stabilizes $x_{\lambda}$ with a domain of attraction estimate $\mathcal{D}_{\lambda}$ and let $\mathcal{S} \subseteq \Lambda_{S}, 0 \in \mathcal{S}$, be such that Assumption 5.1 holds. In addition, assume $\lambda_{\mathcal{S}}(x)$, $x \in \mathcal{D}_{c}$, is such that $V(x), x \in \mathcal{D}_{c}$, given by (7) holds, and, for $x_{0} \in \mathcal{D}_{c}, x(t), t \in \mathcal{I}_{x_{0}}$, is the solution to (2) with the feedback control law $u=\phi_{\lambda_{\mathcal{S}}(x)}(x), x \in \mathcal{D}_{c}$. If $x_{0} \in \mathcal{D}_{c}$, then $x(t) \rightarrow \hat{\mathcal{M}} \triangleq \bigcup_{\gamma \in \mathcal{G}} \mathcal{M}_{\gamma}$ as $t \rightarrow \infty$, where $\mathcal{G} \triangleq\{\gamma \geq$ $\left.0: \mathcal{R}_{\gamma} \cap \mathcal{D}_{0} \neq \varnothing\right\}$. If, in addition, $\mathcal{S}_{0} \triangleq\left\{\lambda \in \mathcal{S}: \mathcal{D}_{\lambda} \cap \mathcal{D}_{0} \neq \varnothing\right\}$ is homeomorphic to $[0, a], a>0$, with $0 \in \mathcal{S}_{0}$ corresponding to $0 \in \mathbb{R}$, or $\mathcal{S}_{0}$ consists of only isolated points, then the zero solution $x(t) \equiv 0$ to (2) is locally asymptotically stable with an estimate of domain of attraction given by $\mathcal{D}_{c}$. Finally, if $\mathcal{D}=\mathbb{R}^{n}$ and there exists $\hat{\lambda} \in \mathcal{S}$ such that the feedback control law $\phi_{\hat{\lambda}}(\cdot)$ globally stabilizes $x_{\hat{\lambda}}$, then the above results are global.

Proof: The result follows from Theorems 3.2, 5.1, 5.2, and the fact that if $\mathcal{S}_{0}$ is homeomorphic to $[0, a], a>0$, with $0 \in \mathcal{S}_{0}$ corresponding to $0 \in \mathbb{R}$, or $\mathcal{S}_{0}$ consists of only isolated points then $\hat{\mathcal{M}} \equiv \mathcal{M}_{0} \equiv\{0\}$ establishing local asymptotic stability of the zero solution $x(t) \equiv 0$ to (2) with an estimate of domain 
of attraction given by $\mathcal{D}_{c}$. Next, without loss of generality, let $\hat{\lambda} \in \mathcal{S}$ be the unique value of $\lambda \in \mathcal{S}$ such that $\mathcal{D}_{\hat{\lambda}}=\mathbb{R}^{n}$. Define $\hat{\mathcal{S}} \triangleq\{\lambda \in \mathcal{S}: p(\lambda)<p(\hat{\lambda})\}$ and $\hat{\mathcal{D}}_{c} \triangleq \bigcup_{\lambda \in \hat{\mathcal{S}}} \mathcal{D}_{\lambda}$ which is a compact positively invariant set. Hence, if $x_{0} \in \hat{\mathcal{D}}_{c}$, it follows from the first part of the theorem that the origin is asymptotically stable, with an estimate of the domain of attraction given by $\hat{\mathcal{D}}_{c}$. Now, global asymptotic stability of the origin is immediate by noting that if $x_{0} \notin \hat{\mathcal{D}}_{c}$, then the forward trajectory of (2) approaches $\hat{\mathcal{D}}_{c}$ in a finite time. If, in fact, $x \notin \hat{\mathcal{D}}_{c}$, then $\lambda_{\mathcal{S}}(x)=$ $\hat{\lambda}$ which implies that for all $x \notin \hat{\mathcal{D}}_{c}$ the feedback control law $u=\phi_{\lambda_{S}(x)}(x), x \in \mathcal{D}_{c}$, stabilizes $x_{\hat{\lambda}}$ and, by Assumption 5.1, $x_{\hat{\lambda}} \in \hat{\mathcal{D}}_{c}$. In this case, it follows from Remark 4.1 that for all $x_{0} \notin \hat{\mathcal{D}}_{c}$ there exists a finite time $T>0$ such that $x(T) \in \hat{\mathcal{D}}_{c}$. Hence, global attraction as well as global asymptotic stability of the origin is established for the respective cases.

Remark 5.1: The switching set $\mathcal{S}$ is quite general in the sense that it can have a hybrid topological structure involving isolated points and closed sets homeomorphic to intervals on the real line. In the special case where the switching set $\mathcal{S}$ consists of only isolated points, the hierarchical switching control strategy given by $u=\phi_{\lambda_{\mathcal{S}}(x)}(x), x \in \mathcal{D}_{c}$, is piecewise continuous. Alternatively, in the special case where the switching set $\mathcal{S}$ is homeomorphic to an interval on $\mathbb{R}$, the hierarchical switching control strategy given by $u=\phi_{\lambda_{\mathcal{S}}(x)}(x), x \in \mathcal{D}_{c}$, is not necessarily continuous. The continuous control case will be discussed in Section VI.

Remark 5.2: In the case where the switching set $\mathcal{S}$ is homeomorphic to an interval on $\mathbb{R}$ and a stabilizing controller $\phi_{0}(\cdot)$ for the origin cannot be obtained, i.e., $c_{0}=0,0 \in \mathcal{D}_{c}$ still holds. Hence, Theorem 5.3 guarantees attraction of the origin if $0 \in \partial \mathcal{D}_{c}$. Alternatively, if $0 \in \stackrel{\circ}{\mathcal{D}}_{c}$, then the origin is asymptotically stable.

Finally, it is important to note that since the hierarchical switching nonlinear controller $u=\phi_{\lambda_{\mathcal{S}}(x)}(x), x \in \mathcal{D}_{c}$, is constructed such that the switching function $\lambda_{\mathcal{S}}(x), x \in \mathcal{D}_{c}$, assures that $V(x), x \in \mathcal{D}_{c}$, defined by (7) is a generalized Lyapunov function with strictly decreasing values at the switching points, the possibility of a sliding mode is precluded with the proposed control scheme. In particular, Theorem 5.2 guarantees that the closed-loop state trajectories cross the boundary of adjacent regions of attraction in the state space in a inward direction. Thus, the closed-loop state trajectories enter the lower potential-valued domain of attraction before subsequent switching can occur. Hence, the proposed nonlinear stabilization framework avoids the undesirable effects of high-speed switching onto an invariant sliding manifold.

\section{EXTENSIONS TO NONLINEAR DYNAMIC COMPENSATION}

In this section, we provide an online procedure for computing the switching function $\lambda_{\mathcal{S}}(x), x \in \mathcal{D}_{c}$, such that (7) holds, by constructing an initial value problem for $\lambda_{\mathcal{S}}(x)$ having a fixedorder dynamic compensator structure. Specifically, we consider a switching set $\mathcal{S}$ diffeomorphic to an interval on the real line $\mathbb{R}$ and assume that the switching function $\lambda_{\mathcal{S}}(x), x \in \mathcal{D}_{c}$, is
$C^{1}$. To present this result, consider the nonlinear controlled dynamical system given by (1) with a nonlinear feedback dynamic controller of the form

$$
\begin{aligned}
\dot{x}_{c}(t) & =F_{c}\left(x(t), x_{c}(t)\right) \quad x_{c}(0)=x_{c 0}, t \in \mathcal{I}_{x_{0}} \\
u(t) & =\phi_{c}\left(x(t), x_{c}(t)\right)
\end{aligned}
$$

where $x_{c}(t) \in \mathcal{C} \subseteq \mathbb{R}^{n_{c}}, t \in \mathcal{I}_{x_{0}}$, is the controller state vector, $\mathcal{C}$ is an open set, $\bar{F}_{c}: \mathcal{D} \times \mathcal{C} \rightarrow \mathbb{R}^{n_{c}}$, and $\phi_{c}: \mathcal{D} \times \mathcal{C} \rightarrow \mathcal{U}$. Note that we do not assume any regularity condition on the mappings $F_{c}(\cdot, \cdot)$ and $\phi_{c}(\cdot, \cdot)$. However, we do assume that the nonlinear feedback controlled dynamical system given by (1), (8), and (9) is such that the solutions of the closed-loop system on $\mathcal{I}_{x_{0}}$ are unique and continuously dependent on the closed-loop system initial conditions.

To construct dynamic controllers of the form (8), (9) we assume that the switching set $\mathcal{S}$ is such that there exists a closed interval $[0, a], a>0$, and a diffeomorphism $\sigma:[0, a] \rightarrow \Lambda_{S}$, such that $\sigma(s) \in \mathcal{S}, s \in[0, a]$, and $\sigma(0)=0$. Furthermore, we assume that $V_{\lambda}(\cdot)$ and $c_{\lambda}$ are $C^{1}$ functions of $\lambda \in \mathcal{S}$. Next, recall that Theorem 5.3 guarantees that the feedback control law $u(x)=\phi_{\lambda_{\mathcal{S}}(x)}(x), x \in \mathcal{D}_{c}$, where $\lambda_{\mathcal{S}}(x), x \in \mathcal{D}_{c}$, is given by (7), locally asymptotically stabilizes the origin of the nonlinear feedback controlled dynamical system (2), and $\mathcal{D}_{c}$ is a subset of the domain of attraction. Furthermore, note that if $x_{0} \in \mathcal{D}_{c} \backslash \mathcal{D}_{0}$ it follows by continuity of the closed-loop trajectories $x(\cdot)$ that there exists a finite time $T_{x_{0}}>0$ such that $x\left(T_{x_{0}}\right) \in \partial \mathcal{D}_{0}$ and $\lambda_{\mathcal{S}}\left(x\left(T_{x_{0}}\right)\right)=0$. Now, define $\lambda_{t} \triangleq \lambda_{\mathcal{S}}(x(t))$ and note that since $\mathcal{S}$ is connected and the set-valued map $\Psi(\cdot)$ is continuous, it follows that $\mathcal{R}_{\gamma}=V^{-1}(\gamma), \gamma \geq 0$, which implies that $V(x(t))$, $t \geq 0$, can be constant on the interval $\left[t_{1}, t_{2}\right] \subseteq\left[0, T_{x_{0}}\right]$ only if $x(t) \in \partial \mathcal{D}_{\lambda_{t}}, t \in\left[t_{1}, t_{2}\right]$, which contradicts the fact that $\dot{V}_{\lambda_{t}}(x(t))<0$. Hence, it follows that $V(x(t)), t \in\left[0, T_{x_{0}}\right]$, is a strictly decreasing function and Theorem 5.2 further implies that $x(t) \in \partial \mathcal{D}_{\lambda_{t}}=V_{\lambda_{t}}^{-1}\left(c_{\lambda_{t}}\right), t \in\left[0, T_{x_{0}}\right]$. Thus,

$$
V_{\lambda_{t}}(x(t))=c_{\lambda_{t}}, \quad t \in\left[0, T_{x_{0}}\right]
$$

relates the state trajectories $x(\cdot)$ to the switching function $\lambda_{\mathcal{S}}(x(\cdot))$. For the statement of the main result of this section the following definitions and proposition are needed. Define

$$
\begin{gathered}
\left.v_{\lambda}(x) \triangleq \frac{\partial\left(c_{\lambda}-V_{\lambda}(x)\right)}{\partial \lambda} \quad w_{\lambda} \triangleq \frac{d \sigma(s)}{d s}\right|_{s=\sigma^{-1}(\lambda)} \\
(x, \lambda) \in \mathcal{D}_{c} \times \mathcal{S}
\end{gathered}
$$

and $Q_{\lambda}(x) \triangleq\left(w_{\lambda} / v_{\lambda}(x) w_{\lambda}\right) V_{\lambda}^{\prime}(x)$ which, as shown in Proposition 6.1 below, is well defined for all $x \in \mathcal{D}_{c} \backslash \mathcal{D}_{0}$ and $\lambda=$ $\lambda_{\mathcal{S}}(x)$. In the case where $x \in \mathcal{D}_{0}$, define $Q_{\lambda}(x) \equiv 0$.

Proposition 6.1: Assume $\sigma:[0, a] \rightarrow \mathcal{S}$ is a diffeomorphism, $\lambda_{\mathcal{S}}: \mathcal{D}_{c} \rightarrow \mathcal{S}$ is a $C^{1}$ function, and $V_{\lambda}(\cdot)$ and $c_{\lambda}$ are $C^{1}$ functions of $\lambda \in \mathcal{S}$. Then $v_{\lambda}(x)$ and $w_{\lambda},(x, \lambda) \in \mathcal{D}_{c} \times \mathcal{S}$, defined in (11), are such that $v_{\lambda_{\mathcal{S}}(x)}(x) w_{\lambda_{\mathcal{S}}(x)} \neq 0$ for all $x \in \mathcal{D}_{c} \backslash \mathcal{D}_{0}$. 
Proof: The result follows by differentiating both sides of (10) and noting that for all $x_{0} \in \mathcal{D}_{c}, \dot{V}_{\lambda_{0}}\left(x_{0}\right)<0, \lambda_{0}=$ $\lambda_{\mathcal{S}}\left(x_{0}\right)$. Specifically, differentiating both sides of (10) yields

$$
v_{\lambda_{t}}(x(t)) \dot{\lambda}_{t}=V_{\lambda_{t}}^{\prime}(x(t)) \dot{x}(t), \quad t \in\left[0, T_{x_{0}}\right] .
$$

Next, let $s(t), t \in\left[0, T_{x_{0}}\right]$, be such that $\lambda_{t}=\sigma(s(t))$, $t \in\left[0, T_{x_{0}}\right]$, and note that (12) evaluated at $t=0$ yields $v_{\lambda_{0}}\left(x_{0}\right) w_{\lambda_{0}} \dot{s}(0)=V_{\lambda_{0}}^{\prime}\left(x_{0}\right) \dot{x}(0)=\dot{V}_{\lambda_{0}}\left(x_{0}\right)<0$. Now, noting that $v_{\lambda_{0}}\left(x_{0}\right) w_{\lambda_{0}}$ and $\dot{s}(0)$ are scalars, it follows that $v_{\lambda_{0}}\left(x_{0}\right) w_{\lambda_{0}} \neq 0, x_{0} \in \mathcal{D}_{c} \backslash \mathcal{D}_{0}$.

Next, we present the main result of this section which provides an online procedure for computing the switching function $\lambda_{\mathcal{S}}(x(t)), t \in \mathcal{I}_{x_{0}}$. Specifically, differentiating both sides of (10) with respect to time yields a Davidenko-type differential equation that defines an initial value problem for the switching function and hence the function $\lambda_{\mathcal{S}}(x(t)), t \in \mathcal{I}_{x_{0}}$, is characterized via a homotopy map.

Theorem 6.1: Assume $\sigma:[0, a] \rightarrow \mathcal{S}$ is a diffeomorphism, $\lambda_{\mathcal{S}}: \mathcal{D}_{c} \rightarrow \mathcal{S}$ is a $C^{1}$ function, and $V_{\lambda}(\cdot)$ and $c_{\lambda}$ are $C^{1}$ functions of $\lambda \in \mathcal{S}$. Then, the solutions $x(t)$ and $\lambda(t), t \in \mathcal{I}_{x_{0}}$, of the closed-loop nonlinear feedback controlled dynamical system

$$
\begin{gathered}
\dot{x}(t)=F\left(x(t), \phi_{\lambda(t)}(x(t))\right) \quad x(0)=x_{0}, t \in \mathcal{I}_{x_{0}} \\
\dot{\lambda}(t)=Q_{\lambda(t)}(x(t)) F\left(x(t), \phi_{\lambda(t)}(x(t))\right) \\
\lambda_{0}=\lambda_{\mathcal{S}}\left(x_{0}\right)
\end{gathered}
$$

are such that $\lambda(t)=\lambda_{\mathcal{S}}(x(t)), t \in \mathcal{I}_{x_{0}}$, or, equivalently, $V_{\lambda(t)}(x(t))=c_{\lambda(t)}, t \in \mathcal{I}_{x_{0}}$.

Proof: The result follows by differentiating both sides of (10) with respect to time and noting that $\dot{\lambda}_{t}=w_{\lambda_{t}} \dot{s}(t)$.

Note that the switching function dynamics characterized by (14) defines a fixed-order dynamic compensator of the form given by (8), (9). Specifically, defining the compensator state as $x_{c}(t) \triangleq \lambda(t)$ so that $n_{c}=q$, the dynamic compensator structure is given by

$$
\begin{gathered}
\dot{x}_{c}(t)=Q_{x_{c}(t)}(x(t)) F\left(x(t), \phi_{x_{c}(t)}(x(t))\right), \\
x_{c}(0)=\lambda_{\mathcal{S}}\left(x_{0}\right), \quad t \in \mathcal{I}_{x_{0}} \\
u(t)=\phi_{x_{c}(t)}(x(t)) .
\end{gathered}
$$

Now, it follows from Theorems 5.3 and 6.1 that for all $x_{0} \in \mathcal{D}_{c}$ the nonlinear feedback controlled dynamical system given by (1), (15), and (16), drives $x_{c}(t)$ to $0 \in \mathcal{S}$ in a finite time $T_{x_{0}}>0$. Note that this result does not violate the assumption of uniqueness of solutions of (15) since $x\left(T_{x_{0}}\right) \in \partial \mathcal{D}_{0}$ at the finite time $T_{x_{0}}>0$ does not correspond to a system equilibrium point. Next, since $\lambda(t)=0, t \geq T_{x_{0}}, x(t)$ reaches $0 \in \mathcal{D}$ asymptotically which guarantees asymptotic stability of the origin with an estimate of the domain of attraction given by $\mathcal{D}_{c}$. Similar arguments hold for global asymptotic stability in the case where $\mathcal{D} \equiv \mathcal{D}_{c} \equiv \mathbb{R}^{n}$.

The compensator dynamics (14) characterize the fastest admissible rate of change of the switching function for which the feedback control (16) maintains stability of the closed-loop system. As discussed in Section I, this quantifies the notion of slow-varying system parameters which has been one of the major shortcomings of gain scheduling practices. It is not surprising to note that this rate is an explicit function of the gradient of the equilibria-dependent Lyapunov functions and the gradient of the domains of attraction estimates with respect to the parameterized equilibrium manifold. Furthermore, the rate of change of the switching function also depends on the gradient of the diffeomorphism evaluated along the switching set $\mathcal{S}$; such a dependence can be used to enforce desirable structural properties of the switching set. For further details see [31].

Finally, to elucidate the hierarchical switching nonlinear controller presented in this section and Section V, we present an algorithm that outlines the key steps in constructing the feedback controller.

Algorithm 6.1: To construct the hierarchical switching feedback control $\phi_{\lambda_{\mathcal{S}}(x(t))}(x(t)), t \geq 0$, perform the following steps.

1) Construct the equilibrium manifold of (1) using $u=$ $\varphi(x, \lambda)$, where $\varphi(\cdot, \cdot)$ is an arbitrary function of $\lambda \in \Lambda_{\circ}$. Use $F(x, \varphi(x, \lambda))=0$ to explicitly define the mapping $\psi(\cdot)$ such that $x_{\lambda}=\psi(\lambda), \lambda \in \Lambda_{\circ}$, is an equilibrium point of (1) corresponding to the parameter value $\lambda$. We note that the above parameterization can be constructed using the approaches given in [22], [23].

2) Construct the set $\Lambda_{S} \subseteq \Lambda_{O}$ such that, for each equilibrium point $x_{\lambda}, \lambda \in \Lambda_{S}$, there exists a stabilizing controller $\phi_{\lambda}(\cdot)$ and an associated domain of attraction $\mathcal{D}_{\lambda}$ corresponding to the level set $c_{\lambda}$ and Lyapunov function $V_{\lambda}(\cdot)$. Here, the controllers $\phi_{\lambda}(\cdot), \lambda \in \Lambda_{S}$, can be obtained using any appropriate standard linear or nonlinear stabilization scheme.

3) Construct the switching set $\mathcal{S} \subseteq \Lambda_{S}$ and a potential function $p: \mathcal{S} \rightarrow \mathbb{R}^{+}$such that Assumption 5.1 is satisfied. In particular,

3a) if $\lambda \in \mathcal{S}$ is an isolated point of $\mathcal{S}$ with corresponding equilibrium point $x_{\lambda}$, then there exists $\lambda_{1} \in \mathcal{S}$ such that $p\left(\lambda_{1}\right)<p(\lambda), x_{\lambda} \in \stackrel{\circ}{\mathcal{D}}_{\lambda_{1}}$;

3b) if $\lambda \in \mathcal{S}$ is an accumulation point of $\mathcal{S}$ then Step 3a) is automatically satisfied if $p(\cdot)$ does not achieve a local minimum at $\lambda$;

3c) if $\lambda, \lambda_{1} \in \mathcal{S}, \lambda \neq \lambda_{1}$, is such that $p(\lambda)=p\left(\lambda_{1}\right)$, then $\mathcal{D}_{\lambda} \cap \mathcal{D}_{\lambda_{1}}=\varnothing$.

4) Given the state-space point $x(t)$ at $t \geq 0$, search for solutions to $V_{\lambda}(x(t))=c_{\lambda}, \lambda \in \mathcal{S}$.

4a) If no solution exists, $\lambda_{\mathcal{S}}(x(t))$ is unchanged.

$4 \mathrm{~b})$ If one solution $\lambda_{1}$ exists and $p\left(\lambda_{1}\right)<p(\lambda)$ then switch $\lambda_{\mathcal{S}}(x(t))$ to $\lambda_{1}$.

4c) If more than one solution exists, repeat Step $4 b$ ) with $\lambda_{1}$ replaced by the solution that minimizes $p(\cdot)$. Note that multiple solutions can be avoided by modifying the $c_{\lambda}$ 's.

5) Construct the hierarchical switching feedback controller $\phi_{\lambda_{\mathcal{S}}(x(t))}(x(t))$ where $\lambda_{\mathcal{S}}(x(t)), x \in \mathcal{D}_{c}$, constructed in Step 4) is such that (7) holds.

Note that the existence of a switching set $\mathcal{S}$ and a potential function $p(\cdot)$ such that Step 3 ) is satisfied, can be guaranteed by modifying Step 4a) as follows: 
4a) Given the state space point $x(t)$ at $t=t_{k} \triangleq k \Delta T$, where $\Delta T>0$ and $k=0,1, \ldots$, search for the solutions of $V_{\lambda}\left(x\left(t_{k}\right)\right) \leq c_{\lambda}, \lambda \in \Lambda_{S}$.

In this case, the switching set $\mathcal{S} \subseteq \Lambda_{S}$ need not be explicitly defined and is computed online. Furthermore, the case where $\Delta T \rightarrow 0$ recovers the continuous framework described in this section. Finally, we note that hierarchical controllers based on the framework in this paper are reported in [18] for controlling rotating stall and surge in axial flow compressors.

\section{CONCLUSION}

A nonlinear control-system design framework predicated on a hierarchical switching controller architecture parameterized over a set of system equilibria was developed. Specifically, a hierarchical switching nonlinear control strategy is constructed to stabilize a given nonlinear system by stabilizing a collection of nonlinear controlled subsystems. The switching nonlinear controller architecture is designed based on a generalized Lyapunov function obtained by minimizing a potential function over a given switching set induced by the parameterized system equilibria. An online procedure for computing the switching scheme was proposed by constructing an initial value problem having a fixed-order dynamic compensator structure.

\section{REFERENCES}

[1] G. Stein, "Adaptive flight control—A pragmatic view," in Application of Adaptive Control, K. S. Narendra and R. V. Monopoli, Eds. New York: Academic, 1980.

[2] W. J. Rugh and J. S. Shamma, "A survey of research on gain scheduling," Automatica, vol. 36, no. 10, 2000.

[3] J. S. Shamma and M. Athans, "Analysis of gain scheduled control for nonlinear plants," IEEE Trans. Automat. Contr., vol. 35, pp. 898-907, Aug. 1990.

[4] D. A. Lawrence and W. J. Rugh, "Gain scheduling dynamic linear controllers for a nonlinear plants," Automatica, vol. 31, pp. 381-390, 1995

[5] J. S. Shamma and M. Athans, "Guaranteed properties of gain scheduled control for linear parameter-varying plants," Automatica, vol. 27, no. 3, pp. 559-564, 1991

[6] - "Gain scheduling: Potential hazards and possible remedies," IEEE Contr. Syst. Mag., vol. 12, pp. 101-107, June 1992.

[7] T. Kuang-Hsuan and J. S. Shamma, "Nonlinear gain-scheduled control design using set-valued methods," in Proc. Amer. Contr. Conf., Philadelphia, PA, 1998, pp. 1195-1199.

[8] M. Jamshidi, North-Holland Series in System Science and Engineering: Large-Scale Systems: Modeling and Control. New York: North-Holland, 1983, vol. 9 .

[9] P. E. Caines and Y.-J. Wei, "Hierarchical hybrid control systems: A lattice theoretic formulation," IEEE Trans. Automat. Contr., vol. 43, pp. 501-508, Apr. 1998.

[10] J. E. R. Cury, B. H. Krogh, and T. Niinomi, "Synthesis of supervisory controllers for hybrid systems based on approximating automata," IEEE Trans. Automat. Contr., vol. 43, pp. 564-568, Apr. 1998.

[11] K. A. Loparo, J. T. Aslanis, and O. Hajek, "Analysis of switched linear systems in the plane-Part 1: Local behavior of trajectories and local cycle geometry," J. Opt. Theory Appl., vol. 52, no. 3, pp. 365-394, 1987.

[12] _ - "Analysis of switched linear systems in the plane-Part 2: Globa behavior of trajectories, controllability and attainability," J. Opt. Theory Appl., vol. 52, no. 3, pp. 395-427, 1987.

[13] P. Peleties and R. A. DeCarlo, "Asymptotic stability of m-switched systems using Lyapunov-like functions," in Proc. Amer. Contr. Conf., Boston, MA, 1991, pp. 1679-1684.
[14] J. Malmborg, B. Bernhardsson, and K. J. Astrom, "A stabilizing switching scheme for multi controller systems," in Proc. 13th IFAC World Congress, San Francisco, CA, 1996, pp. 229-234.

[15] J. Guckenheimer, "A robust hybrid stabilization strategy for equilibria," IEEE Trans. Automat. Contr., vol. 40, pp. 321-326, Feb. 1995.

[16] IEEE Trans. Automat. Contr. (Special Issue), vol. 43, Apr. 1998.

[17] Automatica (Special Issue), vol. 35, no. 3, 1999.

[18] A. Leonessa, V. Chellaboina, and W. M. Haddad, "Globally stabilizing controllers for multi-mode axial flow Ccmpressors via equilibria-dependent Lyapunov functions," in Proc. Amer. Contr. Conf., Albuquerque, NM, 1997, pp. 993-997.

[19] M. W. McConley, B. D. Appleby, M. A. Dahleh, and E. Feron, "A control Lyapunov function approach to robust stabilization of nonlinear systems," in Proc. Amer. Contr. Conf., Albuquerque, NM, 1997, pp. 329-333.

[20] F. H. Clarke, Y. S. Ledyaev, E. D. Sontag, and A. I. Subbotin, "Asymptotic controllability implies feedback stabilization," IEEE Trans. Automat. Contr., vol. 42, pp. 1394-1407, Oct. 1997.

[21] M. Ikeda, Y. Ohta, and D. D. Siljak, "Parametric stability," in New Trends in Systems Theory, G. Conte, A. M. Perdon, and B. Wyman, Eds. Boston, MA: Birkhauser, 1991, pp. 1-20.

[22] Y. Ohta and D. D. Siljak, "Parametric quadratic stabilizability of uncertain nonlinear systems," Syst. Control Lett., vol. 22, no. 6, pp. 437-444, 1994.

[23] H. G. Kwatny and B.-C. Chang, "Constructing linear families from parameter-dependent nonlinear dynamics," IEEE Trans. Automat. Contr. vol. 43, pp. 1143-1147, Aug. 1998

[24] J. K. Hale, Ordinary Differential Equations. Malabar, FL: Krieger, 1980.

[25] E. A. Coddington and N. Levinson, Theory of Ordinary Differential Equations. New York: McGraw-Hill, 1955.

[26] A. F. Filippov, Differential Equations with Discontinuous Right-Hand Sides. Dordrecht, The Netherlands: Kluwer, 1988.

[27] H. K. Khalil, Nonlinear Systems. Englewood Cliffs, NJ: Prentice-Hall, 1996.

[28] H. L. Royden, Real Analysis. New York: Macmillan, 1988.

[29] N. P. Bhatia and G. P. Szegö, Stability Theory of Dynamical Systems. New York: Springer-Verlag, 1970.

[30] J.-P. Aubin, Viability Theory. Boston, MA: Birkhäuser, 1991

[31] A. Leonessa, W. M. Haddad, and V. Chellaboina, "Optimal nonlinear switching control," in Proc. IEEE Conf. Dec. Contr., Phoenix, AZ, 1999, pp. 5158-5163.

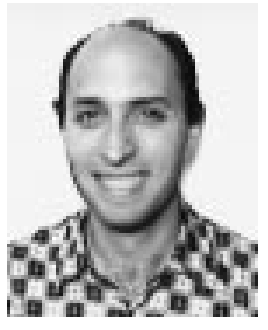

Alexander Leonessa received the B.S. degree in aeronautical engineering in 1993 from the University of Rome "La Sapienza," Rome, Italy, the M.S. degree in aerospace engineering in 1997, and the M.S. degree in applied mathematics and the Ph.D. degree in aerospace engineering in 1999 from Georgia Institute of Technology, Atlanta, with specialization in stability theory for nonlinear dynamical switching systems.

From 1993 to 1994, he served as an Officer in the Italian Navy and in 1995, he joined Alitalia Airlines as an Aircraft Maintenance Supervisor. From 1995 to 1999, he was a Research Assistant in the School of Aerospace Engineering, Georgia Institute of Technology. In 2000, he joined the faculty of the Ocean Engineering Department, Florida Atlantic University, Boca Raton, where he is currently an Assistant Professor. His current research interests are in the areas of stability theory of nonlinear systems, robust control, adaptive control, saturation control, optimal control, nonlinear dynamic compensation, gain scheduling, sliding mode control, linear parameter-varying control, and hierarchical switching control. His applied areas of interest include active control and modeling of propulsion systems, autonomous vehicle control systems, attitude stability and control, and robot control. He is a coauthor (with W. M. Haddad and V. Chellaboina) of $\mathrm{Hi}$ erarchical Nonlinear Switching Control Design with Applications to Propulsion Systems (Springer-Verlag, 2000). 


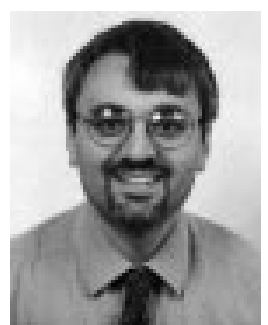

Wassim M. Haddad received the B.S., M.S., and $\mathrm{Ph} . \mathrm{D}$. degrees in mechanical engineering, with specialization in dynamics and control, from Florida Institute of Technology, Melbourne, in 1983, 1984, and 1987 , respectively.

From 1987 to 1994, he served as a Consultant for the Structural Controls Group, Government Aerospace Systems Division, Harris Corporation, Melbourne, FL. In 1988, he joined the faculty of the Mechanical and Aerospace Engineering Department, Florida Institute of Technology, where he founded and developed the Systems and Control Option within the graduate program. Since 1994, he has been a member of the faculty of the School of Aerospace Engineering, Georgia Institute of Technology, Atlanta, where he is currently a Professor. His current research interests are in the areas of nonlinear robust multivariable control for aerospace systems, multiobjective mixed-norm $H_{2} / H_{\infty} H_{2} / L_{1}$, and $H_{\infty} / L_{1}$ controller synthesis, fixed-architecture decentralized control, stability theory for nonlinear systems, saturation control, robust nonlinear feedback control, nonlinear disturbance rejection control, adaptive control for nonlinear uncertain systems, nonlinear hierarchical switching control, optimal switching control, and impulsive and hybrid dynamical systems. His applied areas of interest include vibration control of large flexible structures, noise control, and active control of combustion and propulsion systems. He is a coauthor (with A. Leonessa and V. Chellaboina) of the book Hierarchical Nonlinear Switching Control Design with Applications to Propulsion Systems (Springer-Verlag, 2000).

Dr. Haddad was a recipient of the National Science Foundation Presidential Faculty Fellow Award.

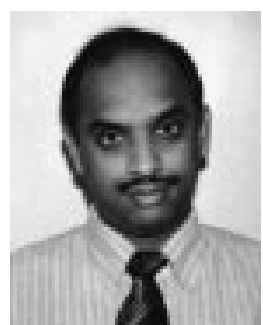

VijaySekhar Chellaboina was born in Andhra Pradesh, India, in 1970. He received the B.Tech. degree in mechanical engineering from Indian Institute of Technology, Madras, India, the M.S. degree in mechanical engineering from Florida Institute of Technology, Melbourne, and the Ph.D. degree in aerospace engineering from Georgia Institute of Technology, Atlanta, with specialization in robust stability analysis and synthesis of linear and nonlinear dynamical systems, in 1991, 1993, and 1996 respectively.

From 1997 to 1999, he was a Research Scientist in the School of Aerospace Engineering, Georgia Institute of Technology. In 1999, he joined the faculty of the Mechanical and Aerospace Engineering Department, University of Missouri, Columbia, where he is currently as Assistant Professor. His current research interests are in the areas of stability theory of nonlinear systems, optimal control, multi-objective control, robust controller analysis and synthesis, linear and nonlinear $H_{\infty}$ theory, robust nonlinear control, dissipative dynamical systems, nonlinear adaptive control, saturation control, switching control, and impulsive and hybrid dynamical systems. His applied areas of interest include control of flexible spacecrafts, flight control systems, active control of propulsion systems, and flow control for aerospace vehicles. 